\title{
Parahydrogen Induced Polarization in Scalar Coupled Systems: Analytical Solutions for Spectral Patterns and their Field Dependence
}

\author{
By Konstantin L. Ivanov ${ }^{1,2}$, Alexandra V. Yurkovskaya ${ }^{1,2}$, and Hans-Martin Vieth ${ }^{3, *}$ \\ ${ }^{1}$ International Tomography Center, Institutskaya 3a, Novosibirsk 630090, Russia \\ 2 Novosibirsk State University, Pirogova 2, Novosibirsk 630090, Russia \\ ${ }^{3}$ Institut für Experimentalphysik, Freie Universität Berlin, Arnimallee 14, D-14195 Berlin, Germany
}

Dedicated to Professor Hans Wolfgang Spiess on the occasion of his $70^{\text {th }}$ birthday

(Received May 4, 2012; accepted in revised form June 27, 2012)

(Published online September 24, 2012)

\section{Spin Hyperpolarization / Para-Hydrogen / PHIP / Magnetic Field Dependence}

We have obtained analytical solutions for Para-Hydrogen Induced Polarization (PHIP) for several types of coupled spin systems, namely, for AB-, ABX-, AA'A"- and $\mathrm{A}_{2} \mathrm{~B}-$ systems. Scalar spin-spin interactions were considered the factor, that determines the PHIP spectral pattern; it is the variation of the spin coupling regime (from strong coupling at low field to weak coupling at high field), which is responsible for the PHIP magnetic field dependence. The field dependence of polarization was considered in detail, general peculiarities of PHIP were found, PHIP patterns were calculated for the systems mentioned. Special attention was paid to the effects of field switching on PHIP.

\section{Introduction}

Para-Hydrogen Induced Polarization (PHIP) represents an important case of strong non-thermal spin polarization or hyperpolarization (HP). HP, in general, arises from non-equilibrium populations of the nuclear spin energy levels and manifests itself in anomalous amplitude and phase of the NMR signals [1-8]. As the intensities of the NMR lines are directly proportional to the population differences for the corresponding transitions (which are tiny at thermal equilibrium) exploiting HP allows one to boost efficiently the sensitivity of NMR spectroscopy. Since sensitivity is frequently the main limitation in NMR applications, development of HP-based NMR techniques is a topical field of research. During the last few years a constant increase of interest in spin hyperpolarization and its applications was observable. PHIP is a particularly promising HP technique, which may find many applications in NMR spectroscopy and imaging as it is known to provide signal enhancements up to several orders of magnitude [911]. A classical way of creating PHIP is catalytical attachment of the para-hydrogen

* Corresponding author. E-mail: hans-martin.vieth@physik.fu-berlin.de 
molecule $\left(\mathrm{H}_{2}\right.$ molecule in its singlet spin state) to a molecule with a double or triple $\mathrm{C}-\mathrm{C}$ bond. Recently, an alternative and rather general way of creating PHIP was provided by reversible interactions with para-hydrogen, where direct hydrogenation is not required [12]. The remarkable NMR enhancement provided by PHIP comes from the fact that at low temperatures the di-hydrogen molecule can be relatively easily enriched in its para-component. Although the para- $\mathrm{H}_{2}$ molecule itself does not give any NMR signal because of chemical (and, consequently, magnetic) equivalence of its two hydrogen nuclei, equivalency removal of the two hydrogen atoms in the hydrogenation reaction products gives rise to their strongly enhanced NMR signals.

Two protocols of performing the PHIP experiments are well documented and referred to as ALTADENA (Adiabatic Longitudinal Transport After Dissociation Engenders Nuclear Alignment) [13] and PASADENA (Parahydrogen and Synthesis Allow Dramatically Enhanced Nuclear Alignment) [14] experiments. In the ALTADENA experiment, first, by a para-hydrogenation reaction at low field (presumably, comparable to the Earth's magnetic field) non-thermal polarization is created in the reaction products. Then the polarized sample is transported into the NMR-spectrometer where the NMR (ALTADENA) spectrum is recorded. In the PASADENA experiment the molecules are polarized inside the NMR spectrometer and the NMR spectrum is detected immediately after running the hydrogenation reaction. In both types of spectra (ALTADENA and PASADENA) line intensities are strongly enhanced as compared to those recorded at thermal equilibrium; however, the appearance of the two spectra is different. In the ALTADENA spectrum the two protons, which constituted the para- $\mathrm{H}_{2}$ molecule prior to the reaction, acquire net HP of opposite sign and equal amplitude. In addition, they exhibit multiplet polarization. In PASADENA spectra the two polarized protons acquire only multiplet HP. Net polarization of the $i$-th spin refers to the z-projection of its magnetization. It is given by the expectation value, $\left\langle I_{i z}\right\rangle$ of the zcomponent of the corresponding spin operator, $\hat{I}_{i z}$. By multiplet polarization of spins $i$ and $j$ we mean their non-equilibrium mutual orientation or entanglement (two-spin order) that is defined as the expectation value of the operator product, $\left\langle I_{i z} I_{j z}\right\rangle$. Higher spin orders for multi-spin systems can be introduced in the same fashion. Both types of polarization can be directly obtained from the NMR spectra of the polarized spin system. The net polarization of a selected spin can be obtained by integrating over all NMR lines corresponding to a transition of this spin, whereas the multiplet HP reveals itself in different line intensities within a given spin multiplet.

The origin of both ALTADENA and PASADENA effects is well understood [13, 14]. In both cases HP appears due (i) to equivalency removal for the pair of protons as a result of the chemical reaction and (ii) to their scalar spin-spin coupling. As a consequence of equivalency removal the two protons give NMR signals at different frequencies, while spin-spin coupling transfers PHIP into NMR observables and determines the spectral pattern. Considering the ALTADENA case the transport of the polarized molecule from low magnetic field to the high field of the NMR-spectrometer is an important step, which results in additional spin evolution and produces net HP of the protons. In general, spin-spin interactions play an essential role in PHIP phenomena because they affect the phase of the PHIP pattern and mediate PHIP transfer to other nuclei. Typically, at low magnetic fields $B$ the spins are coupled strongly (their coupling constant, $J$, is larger or comparable to their difference in Zeeman interaction, $\delta v)$, 
whereas at the high field of the NMR spectrometer they are coupled weakly $(J \ll \delta v)$. The variation of the coupling regime results in the pronounced difference between the ALTADENA and PASADENA spectra. Strong coupling of spins may also result in fast and efficient HP transfer among them proceeding at a rate of approximately $J$.

The main concern of the present work is the theoretical treatment of PHIP phenomena, in particular, obtaining results for the magnetic field dependence of PHIP in several spin systems of different coupling characteristics. So far, the theoretical description of PHIP phenomena has been rather limited. The field dependence of the PASADENA spectra of two-spin $1 / 2$ systems has been considered theoretically in a single publication by Bouget-Bonnet et al. [15]. In that work expressions for different spin orders in PASADENA spectra have been obtained for arbitrary coupling of the two polarized protons, thus the standard limitation of the spin system to the weakly coupled AX-system has been overcome. Theoretical treatment of higher-spin systems is also limited to a few publications. Recently Vinogradov and Grant [16] obtained an analytical solution for ALTADENA of the three-spin 1/2 system. They considered coupled protons and determined the populations of the spin energy levels at zero field and those at high detection field after adiabatic field switching. Jóhannesson et al. [17] considered a three-spin system, in which two nuclei originate from para-hydrogen, while the third spin is a hetero-nucleus. They obtained an analytical solution for non-thermal polarization of the hetero-nucleus in the course of the co-called "diabatic-adiabatic" field cycling. Aime and coauthors [18] treated theoretically a three-spin system and studied the possibility of transferring PHIP to a hetero-nucleus pointing out that its polarization is of multiplet character. Recently the group of H. W. Spiess [19] considered an AA'BB' system analytically and revealed the role of nuclear spin level crossings in the PHIP formation.

Recently we proposed a theoretical approach to the transfer of HP in scalar coupled multispin systems at arbitrary magnetic field [20]. As the type of HP and its preparation rate were taken arbitrary, the method can be applied to the PHIP case. We also discussed PHIP transfer effects among the coupled spins at low field and calculated the PHIP field dependence for three-spin 1/2 systems. Applying this theoretical approach also to describing the PHIP magnetic field dependence of real multispin systems lead to an almost ideal agreement between theory and experiment [21]. These results allowed us to conclude that scalar spin-spin interactions represent the predominant factor responsible for the formation of PHIP and transferring it to other nuclei. Here we will employ the same approach focusing on analytical solutions for PHIP. We will consider coupled spin 1/2 systems of increasing complexity. Systems with higher spins, in particular the analogue of PHIP, Ortho-Deuterium Induced Polarization (ODIP) [22], represent an interesting extension of the present treatment, which is, however, beyond the scope of this work. All the spin systems will be named in accordance with standard NMR terminology applicable to the coupling regime at the polarization field $B_{\mathrm{p}}$. At the detection field $B_{0}$ the spins will always be assumed to be coupled weakly. In particular, we will consider PHIP effects in a coupled 2-spin system (AB-system), an AB-system with weak coupling to a third spin (ABX-system), a 3-spin system with two equivalent spins $\left(\mathrm{A}_{2} \mathrm{~B}\right.$-system) and a very strongly coupled 3 -spin system (AA' $\mathrm{A}^{\prime \prime}$-system). Special attention will be paid to the field dependence of PHIP effects and to the influence of the field switching speed on the PHIP spectra. 
The outline of the article is as follows: In Sect. 2 we will briefly present the theory for PHIP of multi-spin systems. In Sect. 3 we will obtain analytical results for PHIP of AB-, ABX-, $\mathrm{A}_{2} \mathrm{~B}$ - and $\mathrm{AA}^{\prime} \mathrm{A}^{\prime \prime}$-systems at different speed of field variation and analyze different types of spin order in HP. Section 4 summarizes the results of the article.

\section{Theoretical approach to PHIP in multi-spin system at variable magnetic field}

In our theoretical approach to PHIP in multi-spin systems we refer to experiments performed in the following three subsequent steps: (1) PHIP is prepared at the desired field $B_{\mathrm{p}}$ during time $\tau_{\mathrm{p}}$ by attachment of para-hydrogen to the non-polarized molecule (preparation step); (2) the external magnetic field is switched from $B_{\mathrm{p}}$ to the observation field $B$ during time $\tau_{\mathrm{fv}}$ (field variation step); (3) HP spectra are acquired by Fourier transform (FT) NMR at $B=B_{0}$ (detection step). Preparation time $\tau_{\mathrm{p}}$ will be considered long so that all coherences between the spin states are washed out (see text below) at the end of the preparation period. Field variation effects will be taken into account at arbitrary time profile, $B(t)$, and considered in detail for the two limiting cases of adiabatic and sudden field switching. Experimentally such a protocol has been realized, for instance, by means of our fast field-cycling device, where the whole NMR probe is moved between the polarization and detection fields $[23,24]$. It is worth noting that field-cycling is usually a prerequisite for observing the hyperpolarized NMR signals, when PHIP is prepared at low magnetic fields. An exception is given by situations where PHIP is observed directly at zero [25,26] or Earth's [27] or low [28,29] magnetic field. These examples, however, require using specialized experimental techniques for detecting the NMR spectra and will not be considered in our work.

As is typical for liquid samples the behavior of a system of $K$ spins $1 / 2$ in an external magnetic field $B_{\mathrm{p}}$ is described by the following Hamiltonian (in units of $\hbar$ ):

$$
\hat{H}=2 \pi\left\{-\sum_{i=1}^{K} v_{i} \hat{I}_{i z}+\sum_{i<j}^{K} J_{i j}\left(\hat{\mathbf{I}}_{i} \cdot \hat{\mathbf{I}}_{j}\right)\right\},
$$

where $v_{i}=\left(1-\sigma_{i}\right) v_{p}^{i}=\gamma_{i} B_{\mathrm{p}}\left(1-\sigma_{i}\right) / 2 \pi=\gamma_{i} B_{\mathrm{p}}\left(1+\delta_{i}\right) / 2 \pi$ is the Larmor precession frequency of the $i$-th spin determined by its chemical shift $\sigma_{i}$ (here $\gamma_{i}$ is the nuclear gyromagnetic ratio of the $i$-th nucleus), and $J_{i j}$ the scalar spin-spin coupling of the $i$-th and $j$-th spins. For convenience sometimes the $\delta$-scale of chemical shifts is used instead of the $\sigma$-scale; the relation between chemical shifts in the two scales is $\sigma_{i}=-\delta_{i}$. The spins $i$ and $j$ are considered weakly coupled when the nuclear spin-spin interaction between them is much smaller than the difference in their Zeeman interactions with the external field: $\left|J_{i j}\right| \ll\left|v_{i}-v_{j}\right|$, otherwise, they are coupled strongly. Thus, not the absolute, but only the relative strength of the coupling with respect to $\left(v_{i}-v_{j}\right)$ is important for fulfilling the strong coupling condition, which therefore depends on the external magnetic field. Spin relaxation effects will be completely neglected in the present consideration. Here we will only briefly summarize the main formulas used in the theoretical approach and will not go into details, as they have been discussed in a previous publication [21]. 
As mentioned above we will consider only the situation of long preparation times $\tau_{\mathrm{p}}$ so that at the end of the preparation period the density matrix of the spin system in the eigen-basis of the Hamiltonian $\hat{H}$ has only diagonal elements. All off-diagonal elements (coherences) have faded during preparation when $\tau_{\mathrm{p}} \gg J^{-1}$. Such a situation is typical for PHIP since hydrogenation reactions are relatively slow. To observe directly the coherence effects in HP different techniques such as Chemically Induced Dynamic Nuclear Polarization (CIDNP) [1] are more suitable. It allows one to use much shorter preparation times so that $\tau_{\mathrm{p}} \ll J^{-1}$ and coherences between the spin states of the polarized molecules become visible [30-32]. In such case a more elaborate theoretical approach has to be utilized [20]. The presence of coherences is important in low-field experiments with HP because polarization transfer that is caused by strong coupling of spins becomes possible due to the evolution of coherences [20,30,31,33]. Here we will neither consider the time evolution at low field nor coherent effects in HP re-distribution, which reveal themselves in an oscillatory component of the HP transfer kinetics. Thus, only stationary solutions [21] for the density matrix of the polarized spin system will be used and all coherences will be set equal to zero. Initially, the state of the polarized spin system can be described by the following density matrix in the Zeeman basis:

$$
\hat{\sigma}^{0}=\hat{\sigma}_{1,2}^{S} \otimes \prod_{i=3}^{K} \hat{\sigma}_{i}^{e q} .
$$

Here "П" stands for the direct product of matrices. The density matrix $\hat{\sigma}_{1,2}^{S}$, which has only the following four non-zero elements (as written in the Zeeman basis),

$$
\left(\sigma_{1,2}^{S}\right)_{\alpha \beta, \alpha \beta}=\left(\sigma_{1,2}^{S}\right)_{\beta \alpha, \beta \alpha}=\frac{1}{2}, \quad\left(\sigma_{1,2}^{S}\right)_{\alpha \beta, \beta \alpha}=\left(\sigma_{1,2}^{S}\right)_{\beta \alpha, \alpha \beta}=-\frac{1}{2},
$$

describes the spins prepared in their singlet state. Here we assume that spins 1 and 2 are those coming from the para- $\mathrm{H}_{2}$ molecule and will keep this assignment throughout the whole article. As usual, the $|\alpha\rangle$ and $|\beta\rangle$ states are states of the $1 / 2$ spin with zprojections $+1 / 2$ and $-1 / 2$, respectively. Equation (3) implies that prior to the reaction all the $\mathrm{H}_{2}$ molecules are in their singlet state. To take account of incomplete enrichment no additional treatment is needed: it is sufficient to multiply the polarization obtained for fully enriched para-hydrogen by the coefficient $\chi=\left(p_{\text {para }}-p_{\text {ortho }} / 3\right)$ (here $p_{\text {para }}$ and $p_{\text {ortho }}$ are the relative populations of para- and ortho-hydrogen, $\left.p_{\text {para }}+p_{\text {ortho }}=1\right)$. Hereafter we will assume that all the di-hydrogen molecules are in their para-state. The other spins $(i>2)$ in the system are at thermal equilibrium prior to the chemical reaction and their Boltzmann polarization is negligibly small. This situation is described by the following density matrices:

$$
\hat{\sigma}_{i}^{e q}=\left(\begin{array}{ll}
1 / 2 & 0 \\
0 & 1 / 2
\end{array}\right) .
$$

The trace of the density matrix $\hat{\sigma}^{0}$ defined by Eqs. (2)-(4) is equal to unity. In the eigenbasis of the spin Hamiltonian (1) the density matrix of the spin system is as follows:

$$
\hat{\rho}^{0}=\hat{T}^{-1} \hat{\sigma}^{0} \hat{T}
$$


with $\hat{T}$ being the matrix composed of the eigen-vectors of the Hamiltonian $\hat{H}$. At the end of the preparation period the population $p_{i}$ of the $i$-th state is equal to the corresponding diagonal element of $\hat{\rho}^{0}$

$$
p_{i}\left(t=\tau_{\mathrm{p}}\right)=\rho_{i i}^{0},
$$

while the coherences between the eigen-states of the spin system are equal to zero. Thus, at the end of the preparation period the spin density matrix, $\hat{\rho}$, is diagonal in the eigen-basis:

$$
\hat{\rho}_{i j}=\delta_{i j} p_{i}=\delta_{i j} \rho_{i i}^{0},
$$

with $\delta_{i j}=1$ for $i=j$ and 0 for $i \neq j$, while in the Zeeman basis it is of the form

$$
\hat{\sigma}=\hat{T} \hat{\rho} \hat{T}^{-1} \text {. }
$$

The density matrix obtained is later changed upon field variation and application of the detecting RF-pulse. It is well-known that in the presence of HP field variation has a considerable effect on the NMR spectra taken at high field. In order to take it into account one has to solve the Liouville equation for the density matrix $\hat{\sigma}$ with the time-dependent Hamiltonian $\hat{H}(t)$ :

$$
\partial_{t} \hat{\sigma}=-i[\hat{H}(t), \hat{\sigma}], \quad t \in\left[\tau_{\mathrm{p}} ; \tau_{\mathrm{p}}+\tau_{\mathrm{fv}}\right] .
$$

The Hamiltonian

$$
\hat{H}(t)=2 \pi\left\{-\sum_{i=1}^{K} v_{i}(t) \hat{I}_{i z}+\sum_{i<j}^{K} J_{i j}\left(\hat{\mathbf{I}}_{i} \cdot \hat{\mathbf{I}}_{j}\right)\right\}
$$

depends on time since the precession frequencies of spins

$$
v_{i}(t)=\gamma_{i} B(t)\left(1-\sigma_{i}\right) / 2 \pi
$$

change upon field variation as $B(t)$ goes from $B_{\mathrm{p}}$ to $B_{0}$. In general, field variation results in exchange of populations among the coupled spin states. Such a mixing of populations can occur only for states characterized by the same $\left\langle I_{z}\right\rangle$ value (expectation value of the z-projection of the total spin operator, $\left.\hat{I}_{z}=\sum_{i} \hat{I}_{i z}\right)$ because $\left[\hat{I}_{z}, \hat{H}(t)\right]=0$ at any instant of time $t$. We will consider in detail only the two limiting cases of field switch: adiabatic variation and sudden field jump. In the adiabatic regime, the changes of the Hamiltonian are so slow that the initial state populations follow the corresponding eigen-states, which change upon field variation. This means that within a manifold with fixed $I_{z}$ value one can correlate the high-field states at $B_{0}$ with the corresponding low-field states at $B_{\mathrm{p}}$. The state of the lowest energy at $B_{0}$ will then have the same population as that at $B_{\mathrm{p}}$, analogously, the population of the second lowest state at $B_{0}$ is the same as that of the second lowest state at $B_{\mathrm{p}}$ and so on. Sudden field switching implies that time $\tau_{\mathrm{fv}}$ is so short that spin evolution has no time to proceed during the field variation step. Therefore the spin density matrix at $t=\tau_{\mathrm{p}}+\tau_{\mathrm{fv}}$ is equal to $\hat{\sigma}\left(t=\tau_{\mathrm{p}}\right)$. If the speed of field variation 
is arbitrary it may become necessary to solve numerically equation (9) with realistic time profiles $B(t)$ in order to simulate experimental results. A simple estimate for the minimal time $\tau_{\mathrm{fv}}$ for adiabatic field switching is given by the condition $\tau_{\mathrm{fv}} \geq 1 / J_{i j}$. However, one should keep in mind that this estimate can be very rough if the magnetic field does not change linearly with time or, most notably, if there are anti-crossings of the nuclear spin levels in the field range between $B_{\mathrm{p}}$ and $B_{0}$. Fast field variation in the vicinity of such anti-crossings may result in a breakdown of adiabaticity and in the exchange of populations between the crossing levels. In our analytical treatment we will consider the model systems in both limiting regimes of field variation, adiabatic and sudden. To describe the experimental data on a quantitative level it is necessary [21] to take the actual profile, $B(t)$, of field variation.

To describe the shape of the PHIP spectra we will consider the usual phase-sensitive NMR detection of the FID assuming that a hard, non-selective rf-pulse is applied with its $B_{1}$ field being parallel to the $x$-axis of the rotating frame so that all transitions of the coupled spins are excited. Whereas for weakly coupled spin systems in thermal equilibrium the intensity of all spectral lines varies as $\sin \varphi$ of the pulse rotation angle $\varphi=\gamma B_{1} \tau$, the situation is much more complex for hyperpolarized systems. Under the influence of the pulse the density matrix changes from its starting value $\hat{\sigma}\left(\tau_{\mathrm{p}}+\tau_{\mathrm{fv}}\right)$ to the following one:

$$
\hat{\sigma}^{\prime}=\exp \left(-i \varphi \hat{I}_{x}\right) \hat{\sigma}\left(\tau_{\mathrm{p}}+\tau_{\mathrm{fv}}\right) \exp \left(i \varphi \hat{I}_{x}\right) .
$$

To determine the shape of the spectrum it is necessary [34] to calculate the elements of $\hat{\sigma}^{\prime}$ and the $\hat{I}_{+}=\hat{I}_{y}+i \hat{I}_{x}$ operator in the eigen-basis of the spin Hamiltonian at the detection field:

$$
\hat{H}_{0}=2 \pi\left\{-\sum_{i=1}^{K} \nu_{i}^{0} \hat{I}_{i z}+\sum_{i<j}^{K} J_{i j}\left(\hat{\mathbf{I}}_{i} \cdot \hat{\mathbf{I}}_{j}\right)\right\}, \quad v_{i}^{0}=\gamma_{i} B_{0}\left(1-\sigma_{i}\right) / 2 \pi .
$$

The complex NMR spectrum, $S(v)$, can be calculated as follows [34]:

$$
S(v) \propto \sum_{i, j} \frac{\left(I_{+}\right)_{i j} \sigma_{j i}^{\prime}}{2 \pi i \Delta v_{i j}+1 / T_{2}},
$$

where $\Delta v_{i j}=v-v_{i j}$ is the offset with respect to the center of the resonance. For hard pulses the condition $\gamma_{i} B_{1} / 2 \pi \gg \Delta v_{i j}$ is fulfilled at any $i$ and $j$. Here $v_{i j}=\left(e_{i}-e_{j}\right)$ is given by the eigen-values, $e_{i}$ and $e_{j}$, of the Hamiltonian $\hat{H}_{0}$. In Eq. (14) the matrix elements of $\hat{I}_{+}$and $\hat{\sigma}^{\prime}$ are those calculated in the eigen-basis of $\hat{H}_{0}$. For simplicity we assumed here that the transverse relaxation time, $T_{2}$, is the same for all lines in the spectrum giving the same homogeneous broadening of all NMR lines. Only for small flip angles the FT-NMR spectrum of the polarized spin system is identical with the slowpassage cw-spectrum. Interestingly, the line intensity $I_{\mu \nu}$ does not only depend on the population difference $\left(p_{\mu}-p_{v}\right)$ but also on the population differences of the so-called 'parallel' NMR transitions [34-36]. Also, different spin orders contribute differently to the FT-NMR spectrum: contributions of net polarizations, i.e. of single-spin order, $\left\langle I_{i z}\right\rangle$, are proportional to $\sin \varphi$, those of the two-spin order, $\left\langle I_{i z} I_{j z}\right\rangle$, to $\sin 2 \varphi$ and so 
on $[37,38]$. For a selective pulse, which flips only the $i$-th spin, a similar formula for the spectrum can be written. Only the operator $\hat{I}_{+}=\hat{I}_{y}+i \hat{I}_{x}$ for the total spin has to be replaced by that for the corresponding spin, $\hat{I}_{i+}$. Such a situation is met when soft pulses are used so that for the NMR transitions of a selected spin (or a group of spins) we have $\gamma_{i} B_{1} / 2 \pi \gg \Delta v_{i j}$, whereas for transitions of all other spins the relation is opposite: $\gamma_{i} B_{1} / 2 \pi \ll \Delta v_{i j}$.

The theoretical approach presented above is applicable to scalar coupled spin systems with an arbitrary number of spins. Here we will obtain analytical results for a number of simple spin systems. These results are presented in the following section.

\section{Analytical results for two- and three-spin systems}

First we will treat the following case:

(a) A two-spin 1/2 system polarized at arbitrary magnetic field (AB-system). Two protons coming from para- $\mathrm{H}_{2}$ become non-equivalent in products of the hydrogenation reaction and form an $\mathrm{AB}$-system. We will consider PHIP preparation at arbitrary magnetic field and describe the effects of field switching from $B_{\mathrm{p}}$ to the high detection field. The field dependence of polarization, too, will be calculated.

A similar treatment will be presented also for the following three-spin systems:

(b) ABX three-spin 1/2 system: the third spin is only weakly coupled to spins 1 and 2, which belonged to para- $\mathrm{H}_{2}$ prior to the hydrogenation reaction;

(c) three-spin $1 / 2$ system polarized at very low magnetic field where the difference in Zeeman interactions of the spins with the external field is negligible ( $\mathrm{AA}^{\prime} \mathrm{A}^{\prime \prime}$ system);

(d) $\mathrm{A}_{2} \mathrm{~B}$-system: spins 1 and 2, which formed para- $\mathrm{H}_{2}$ prior to the reaction constitute an AB-system, while spin 3 (initially non-polarized) is equivalent to spin 1 (the second A-spin).

As mentioned above we neglect spin relaxation and assume that all coherences at the polarization field have faded. For the sake of simplicity, we will assume that at the detection field all protons are weakly coupled, although this assumption is by no means a principal limitation of the approach. The two limiting cases of adiabatic and sudden field variation will be considered; the effect of field switching on the PHIP spectral pattern will be analyzed. The spin systems will be discussed in the order of growing complexity: the AB-system represents the simplest case, while addition of the third spin results in PHIP re-distribution in the molecule due to $J$-couplings. As a consequence, overpopulation of the singlet state of $\mathrm{H}_{2}$ is transferred into different polarization order and results in non-thermal polarization of the third spin.

The present treatment is limited to two- and three-spin systems. In principle, the Hamiltonian of systems of more spins $1 / 2$ (e.g., $\left.\mathrm{AA}^{\prime} \mathrm{XX}^{\prime}\right)$ can in certain cases also be solved analytically with expressions for PHIP obtainable. However, these results are already rather cumbersome and cannot be easily analyzed. As the complexity of spin system grows it becomes necessary to solve the problem by numerical methods, which have proven their high accuracy [21]. On the other hand, analytical solutions have their own value as they can reveal the main peculiarities of PHIP patterns, demonstrate effects of field switching and help to understand the conditions, under which PHIP is efficiently transferred to other nuclei. In some cases restricting consideration of the real 
multi-spin system to a two-spin or three-spin system can be an accurate approximation, e.g., when the entire coupled spin system splits into subparts with sufficiently weak coupling among the subsystems that does not reveal itself on the time scale of the experiment. This is given when the coupling of the subparts of the spin system is much smaller than the inverse preparation time, $1 / \tau_{\mathrm{p}}$.

\subsection{Two-spin system of type $\mathrm{AB}$ at the polarization field}

Let us first examine a two-spin system polarized by PHIP at an arbitrary magnetic field $B_{\mathrm{p}}$. The results for such a system have been obtained already for the conditions of PASADENA and ALTADENA (i.e., $B_{\mathrm{p}}=0$ and adiabatic field switching) [13,14]. Here we consider the full field dependence of the PHIP phenomena. Varying the field is a tool for optimizing the net and multiplet HP, i.e., for manipulating the PHIP spectral pattern. In addition, effects of field switching speed will be studied in some detail; comparison of the cases of adiabatic and sudden field variation will be performed. Once field switching becomes non-adiabatic the established results for ALTADENA are no longer valid even when the polarization field is zero. This acronym implies that field variation is adiabatic and thus should be restricted to this case. For fast switching the theoretical treatment of the problem has to be revisited. Such a revision has some practical value because in some experiments it is desirable to have fast (close to sudden) field switching in order to minimize HP losses caused by spin relaxation. Thus, both factors, i.e., magnetic field dependence and field switch, are of importance and will be analyzed analytically in detail. Formulas will be obtained for the net and multiplet HP, while field switching will be considered in the two limiting regimes of adiabatic and sudden field variation. We want to mention that theoretical and experimental results obtained for PASADENA spectra taken at arbitrary field (including the case of strongly coupled spins, i.e., AB-system at the detection field) have already been published [15]. However, these results cannot be directly applied to the case under study as we deal with weakly coupled spins at $B=B_{0}$ and vary the PHIP preparation field $B_{\mathrm{p}}$.

Now let us derive the expressions for the quantities of interest. Spin evolution of the AB-system at $B=B_{\mathrm{p}}$ is described by the following Hamiltonian:

$$
\hat{H}_{\mathrm{AB}}=2 \pi\left\{-v_{1} \hat{I}_{1 z}-v_{2} \hat{I}_{2 z}+J_{12}\left(\hat{\mathbf{I}}_{1} \cdot \hat{\mathbf{I}}_{2}\right)\right\} .
$$

The system of two spins $1 / 2$ has the following eigen-functions at arbitrary magnetic field:

$$
\begin{aligned}
& |1\rangle=|\alpha \alpha\rangle, \quad|2\rangle=\cos \theta|\alpha \beta\rangle+\sin \theta|\beta \alpha\rangle, \quad|3\rangle=-\sin \theta|\alpha \beta\rangle+\cos \theta|\beta \alpha\rangle, \\
& |4\rangle=|\beta \beta\rangle .
\end{aligned}
$$

Here $\theta=\frac{1}{2} \arctan \left(\frac{J}{\left(\sigma_{1}-\sigma_{2}\right) \nu_{p}}\right)$ is the mixing angle of the $|\alpha \beta\rangle$ and $|\beta \alpha\rangle$ states, a key parameter determining the eigen-states of the two-spin system. For clarity, we assume that $\sigma_{1}>\sigma_{2}$, so that $\theta$ falls into the range $[0 ; \pi / 4]$ for positive $J_{12}$ and into the range $[0 ;-\pi / 4]$ for negative $J_{12}$. The mixing angle is field dependent and varies 
monotonously from $\pm \pi / 4$ to zero as the magnetic field $B_{\mathrm{p}}$ goes from zero to $B_{0}$. At the end of the preparation period the populations of the eigen-states are

$$
p_{1}=p_{4}=0, \quad p_{2}=\frac{1-\sin 2 \theta}{2}, \quad p_{3}=\frac{1+\sin 2 \theta}{2} .
$$

When the magnetic field is switched adiabatically and the system is weakly coupled at the detection field (AX-system) and $\sigma_{1}>\sigma_{2}$, the population of state $|2\rangle$ goes to that of $|\alpha \beta\rangle$, while the population of state $|3\rangle$ is identical to that of $|\beta \alpha\rangle$ at the high detection field. Then the net magnetizations of spins are as follows:

$$
\left\langle I_{1 z}\right\rangle=\operatorname{Tr}\left\{\hat{I}_{1 z} \hat{\rho}\left(\tau_{\mathrm{p}}+\tau_{\mathrm{fv}}\right)\right\}=-\frac{\sin 2 \theta}{2}=-\left\langle I_{2 z}\right\rangle=\operatorname{Tr}\left\{\hat{I}_{2 z} \hat{\rho}\left(\tau_{\mathrm{p}}+\tau_{\mathrm{fv}}\right)\right\} .
$$

The multiplet polarization $\left\langle I_{1 z} I_{2 z}\right\rangle$ is field independent and equal to $-1 / 4$. Thus, if $J_{12}<0$ the spin with the largest chemical shift on the $\sigma$-scale (as we have assumed $\left.\left(\sigma_{1}-\sigma_{2}\right)>0\right)$ acquires negative net polarization. However, if $J_{12}$ is negative (i.e., $\theta$ changes sign) the polarizations $\left\langle I_{1 z}\right\rangle$ and $\left\langle I_{2 z}\right\rangle$ will change their sign but keep the same magnitude. Similar effects of the sign of $J$-coupling on the sign of net polarization are also known for other HP techniques at low field, in particular, for CIDNP [39,40].

In the case of sudden field switching the expectation values of all operators do not change upon field variation. Therefore, their values can be calculated as follows from those at the polarization field at the end of the preparation period:

$$
\left\langle I_{1 z}\right\rangle=p_{2}\left\langle 2\left|\hat{I}_{1 z}\right| 2\right\rangle+p_{3}\left\langle 3\left|\hat{I}_{1 z}\right| 3\right\rangle=-\frac{\sin 4 \theta}{4}=-\left\langle I_{2 z}\right\rangle
$$

As in the case of the adiabatic field switching, the multiplet polarization $\left\langle I_{1 z} I_{2 z}\right\rangle$ is field independent and equal to $-1 / 4$.

The dependences of the spin polarization on the external magnetic field strength are shown in Fig. 1. For adiabatic field variation net polarization goes gradually down with the field showing a transition from the ALTADENA case (strong coupling at the polarization field, $\left\langle I_{1 z}\right\rangle=-\left\langle I_{2 z}\right\rangle=-1 / 2$ ) to the PASADENA case (weak coupling at the polarization field, $\left.\left\langle I_{1 z}\right\rangle=\left\langle I_{2 z}\right\rangle=0\right)$. When field switching is sudden the situation is different. Since at zero and high field both quantities $\left\langle I_{1 z}\right\rangle$ and $\left\langle I_{2 z}\right\rangle$ are equal to zero, the field dependence of net HP is a curve with its maximum of $1 / 4$ reached at the magnetic field, at which $\sin 4 \theta=1$, i.e., $\theta=\pi / 8$ and $J=\left(\sigma_{1}-\sigma_{2}\right) v_{p}$. Thus, the results for the two limiting cases of field variation are strongly different, which is an indication that field variation speed is an important factor in low-field PHIP. At very low field the difference between the two limiting cases is most pronounced, since there is maximal net PHIP for the adiabatic regime and zero net PHIP for the sudden field jump. When going to high fields (small $\theta$ angles) at preparation net PHIP becomes smaller taking the same value for both regimes of field switching: $\left\langle I_{1 z}\right\rangle=-\left\langle I_{2 z}\right\rangle \approx-\theta$.

A set of spectra calculated for different $B_{\mathrm{p}}$ and both switching regimes is shown in Fig. 2. At low fields the patterns are quite different for fast and slow field variation, but they exactly coincide at high fields because of the weak coupling of spins. It is interesting to note that the PHIP spectrum at $B_{\mathrm{p}}=0$ and sudden field switching coincides with the PASADENA spectrum at high $B_{\mathrm{p}}$. The reason for such a coincidence is that without 


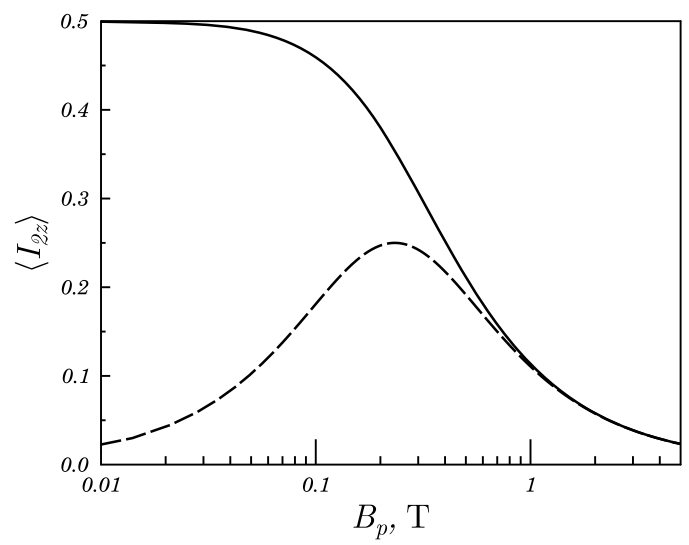

Fig. 1. Magnetic field dependence of PHIP of a 2-spin AB-system. Here only positive net polarization is shown (in this case this is $\left\langle I_{2 z}\right\rangle$; its counterpart, $\left\langle I_{1 z}\right\rangle$, differs only in sign, multiplet PHIP is always equal to $-1 / 4)$; solid line - adiabatic, dashed line - sudden field variation. NMR parameters: $\sigma_{1}=2 \mathrm{ppm}, \sigma_{2}=$ $1 \mathrm{ppm}, J_{12}=10 \mathrm{~Hz}, \chi=\left(p_{\text {para }}-p_{\text {ortho }} / 3\right)=1$.

(a)

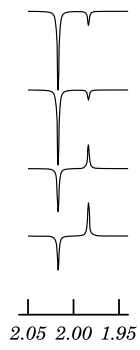

$\sigma, \mathrm{ppm}$ (b)

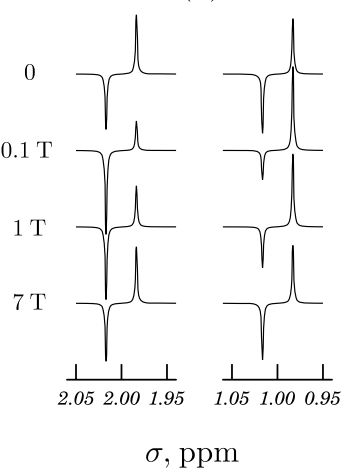

Fig. 2. PHIP spectra of an AB-system for $B_{\mathrm{p}}$ of $0,0.1 \mathrm{~T}, 1 \mathrm{~T}, 7 \mathrm{~T}$; for adiabatic (a) and sudden (b) field variation, pulse rotation angle $\varphi=\pi / 4$. NMR parameters are the same as for Fig. 1 .

an external field net HP of any spin can only be zero, hence, only multiplet polarization $\left\langle I_{1 z} I_{2 z}\right\rangle$ exists. When the field switching is sudden net HP is zero also at the detection field, thus the spectrum must coincide with the PASADENA spectrum. Any non-zero net HP observed in ALTADENA experiments is solely due to the spin dynamics in the slowly varying magnetic field $B(t)$ during the field variation [13,40]. Previously [41] it was proposed that the spectra obtained for the ALTADENA protocol can exhibit the PASADENA like pattern due to the exchange of the polarized protons in the reaction products. Consequently, the population can be evenly distributed between the states $|2\rangle$ and $|3\rangle$. Such an effect was presumably observed by Haake et al. [42]. In our case the population of states $|2\rangle$ and $|3\rangle$ is mixed due to the sudden field switching, which leads to the same PHIP pattern. PHIP spectra on the $\sigma$-scale and the $\delta$-scale of chemical shift 
are mirror images of each other: for $J_{12}>0$ the spin with largest $\sigma_{\mathrm{i}}$ has negative net PHIP, whereas on the $\delta$-scale it is the one with smallest $\delta_{\mathrm{i}}$. One should also note that when non-selective RF pulses are used the shape of the PHIP spectra strongly depends on the pulse rotation angle $\varphi$. For instance, spectra taken at $\varphi=\pi / \underline{4}$ and $\varphi=\pi / 2$ (not shown here) are considerably different as in the latter all contributions of the multiplet polarization vanish.

Now let us demonstrate the changes imposed on the PHIP patterns by addition of a third spin $1 / 2$. In the simplest case the third spin is only weakly coupled to the first two. This is the case of the ABX-system presented in Sect. 3.2.

\subsection{Three-spin system of type $\mathrm{ABX}$ at the polarization field}

The spin system of the ABX type is of great interest because $\mathrm{X}$ can represent a heteronucleus. The possibility to polarize a spin $1 / 2$ hetero-nucleus with long relaxation times is considered to be one of the most promising applications of PHIP [18,43-47]. To obtain net polarization of the $\mathrm{X}$ nucleus resulting from strong coupling of spins it is necessary to go to very low fields, which is rather difficult to achieve [17,47]. Nevertheless, even when the $\mathrm{X}$ nucleus is only weakly coupled to spins 1 and 2 , which constituted the para- $\mathrm{H}_{2}$ molecule prior to the chemical reaction, the $\mathrm{X}$-spin can in certain cases acquire PHIP of multiplet character $[9,18]$. Here we will study this phenomenon in more detail and will obtain simple analytical formulas for PHIP in the ABX-system.

A three-spin $1 / 2$ system becomes an ABX-system when $\left|v_{1}-v_{3}\right|,\left|v_{2}-v_{3}\right| \gg$ $J_{13}, J_{23}$, i.e., spin 3 is always only weakly coupled to spins 1 and 2 . Spin evolution of such a system at the polarization field can be described by the following Hamiltonian:

$$
\hat{H}_{\mathrm{ABX}}=2 \pi\left\{-v_{1} \hat{I}_{1 z}-v_{2} \hat{I}_{2 z}-v_{3} \hat{I}_{3 z}+J_{12}\left(\hat{\mathbf{I}}_{1} \cdot \hat{\mathbf{I}}_{2}\right)+J_{13} \hat{I}_{1 z} \hat{I}_{3 z}+J_{23} \hat{I}_{2 z} \hat{I}_{3 z}\right\} .
$$

This form of the Hamiltonian guarantees that the z-projection of spin $3,\left\langle I_{3 z}\right\rangle$, is always conserved and the eigen-states of the system are split into two groups, one with $\left\langle I_{3 z}\right\rangle=$ $1 / 2$

$$
\begin{aligned}
& |1\rangle_{+}=|\alpha \alpha \alpha\rangle, \quad|2\rangle_{+}=\cos \theta_{+}|\alpha \beta \alpha\rangle+\sin \theta_{+}|\beta \alpha \alpha\rangle, \\
& |3\rangle_{+}=-\sin \theta_{+}|\alpha \beta \alpha\rangle+\cos \theta_{+}|\beta \alpha \alpha\rangle, \quad|4\rangle_{+}=|\beta \beta \alpha\rangle
\end{aligned}
$$

and a second one with $\left\langle I_{3 z}\right\rangle=-1 / 2$

$$
\begin{aligned}
& |1\rangle_{-}=|\alpha \alpha \beta\rangle, \quad|2\rangle_{-}=\cos \theta_{-}|\alpha \beta \beta\rangle+\sin \theta_{-}|\beta \alpha \beta\rangle \\
& |3\rangle_{-}=-\sin \theta_{-}|\alpha \beta \beta\rangle+\cos \theta_{-}|\beta \alpha \beta\rangle, \quad|4\rangle_{-}=|\beta \beta \beta\rangle .
\end{aligned}
$$

The state populations are

$$
p_{1_{ \pm}}=p_{4_{ \pm}}=0, \quad p_{2_{ \pm}}=\frac{1-\sin 2 \theta_{ \pm}}{4}, \quad p_{3_{ \pm}}=\frac{1+\sin 2 \theta_{ \pm}}{4} .
$$

The mixing angles for the two groups of states characterized by different z-projections of spin 3 are:

$$
\theta_{ \pm}=\frac{1}{2} \arctan \left(\frac{J_{12}}{\left(\sigma_{1}-\sigma_{2}\right) v_{p} \pm\left(J_{13}-J_{23}\right) / 2}\right)=\frac{1}{2} \arctan \left(\frac{J_{12}}{\delta v_{ \pm}}\right) .
$$


In the adiabatic case the correlation of states at the polarization and the detection field (where all three spins are assumed to be weakly coupled) is essentially the same as for the AB-system. In the same fashion, the spin states should be correlated separately for both groups corresponding to the fixed states of the $\mathrm{X}$-nucleus. For clarity we assume $\sigma_{1}>\sigma_{2}$ and obtain:

$$
\begin{aligned}
& |2\rangle_{+} \rightarrow|\alpha \beta \alpha\rangle, \quad|3\rangle_{+} \rightarrow|\beta \alpha \alpha\rangle, \\
& |2\rangle_{-} \rightarrow|\alpha \beta \beta\rangle, \quad|3\rangle_{-} \rightarrow|\beta \alpha \beta\rangle .
\end{aligned}
$$

Then the net polarizations of spins at the detection field are

$$
\left\langle I_{1 z}\right\rangle=-\frac{\sin 2 \theta_{+}+\sin 2 \theta_{-}}{4}=-\left\langle I_{2 z}\right\rangle, \quad\left\langle I_{3 z}\right\rangle=0 .
$$

In this expression $\left\langle I_{1,2 z}\right\rangle$ represent net HP from Eq. (18) averaged over the states of the $\mathrm{X}$-spin. Net HP of the X-spin is zero independent of the polarization field and switching speed. For the second-order multiplet polarization we obtain

$$
\left\langle I_{1 z} I_{2 z}\right\rangle=-\frac{1}{4}, \quad\left\langle I_{1 z} I_{3 z}\right\rangle=-\frac{\sin 2 \theta_{+}-\sin 2 \theta_{-}}{8}=-\left\langle I_{2 z} I_{3 z}\right\rangle,
$$

while the third-order multiplet effect, $\left\langle I_{1 z} I_{2 z} I_{3 z}\right\rangle$, is equal to zero.

In the case of sudden field variation the expectation values of all operators can be calculated at the polarization field giving us the net polarizations of spins:

$$
\left\langle I_{1 z}\right\rangle=-\frac{\sin 4 \theta_{+}+\sin 4 \theta_{-}}{8}=-\left\langle I_{2 z}\right\rangle, \quad\left\langle I_{3 z}\right\rangle=0 .
$$

In this expression polarization $\left\langle I_{1,2 z}\right\rangle$ is the average of net HP from Eq. (19) over the state of the $\mathrm{X}$-spin. The second-order multiplet polarizations are

$$
\left\langle I_{1 z} I_{2 z}\right\rangle=-\frac{1}{4}, \quad\left\langle I_{1 z} I_{3 z}\right\rangle=-\frac{\sin 4 \theta_{+}-\sin 4 \theta_{-}}{16}=-\left\langle I_{2 z} I_{3 z}\right\rangle,
$$

while $\left\langle I_{1 z} I_{2 z} I_{3 z}\right\rangle=0$.

Here it is important to note that PHIP does not allow one to transfer net polarization to spin 3 because $\hat{I}_{3 z}$ is preserved as $\left[\hat{I}_{3 z}, \hat{H}_{\mathrm{ABX}}\right]=0$. Nevertheless, its multiplet polarization can be formed efficiently $[9,18]$. This happens only when (i) spins 1 and 2 are strongly coupled and (ii) their difference in spin-spin coupling with spin 3, $\delta J=\left(J_{13}-J_{23}\right)$, is non-zero. At fields so high that $\left|v_{1}-v_{2}\right| \gg\left|J_{13}-J_{23}\right|$ (i.e., where the ABX-system is a weakly coupled AMX-system) this effect vanishes independent of the field variation speed. The reason for the multiplet-type HP of the X-spin is as follows. The degree of net polarization of spin 1 (the same applies to spin 2) is different depending on whether spin 3 is in its $|\alpha\rangle$ or $|\beta\rangle$ state. This is due to the fact that $\delta J \neq 0$ and that $\left\langle I_{1 z}\right\rangle$ and $\left\langle I_{2 z}\right\rangle$ depend on the mixing angle. This means that there is mutual entanglement of spins 1 and 3 , which leads to a non-zero expectation value of $\left\langle I_{1 z} I_{3 z}\right\rangle$. At the same time, when $\left\langle\left(I_{1 z}+I_{2 z}\right) I_{3 z}\right\rangle=0$ the two multiplet polarizations, $\left\langle I_{1 z} I_{3 z}\right\rangle$ and $\left\langle I_{1 z} I_{2 z}\right\rangle$, must have the same amplitude and opposite sign. This consideration 


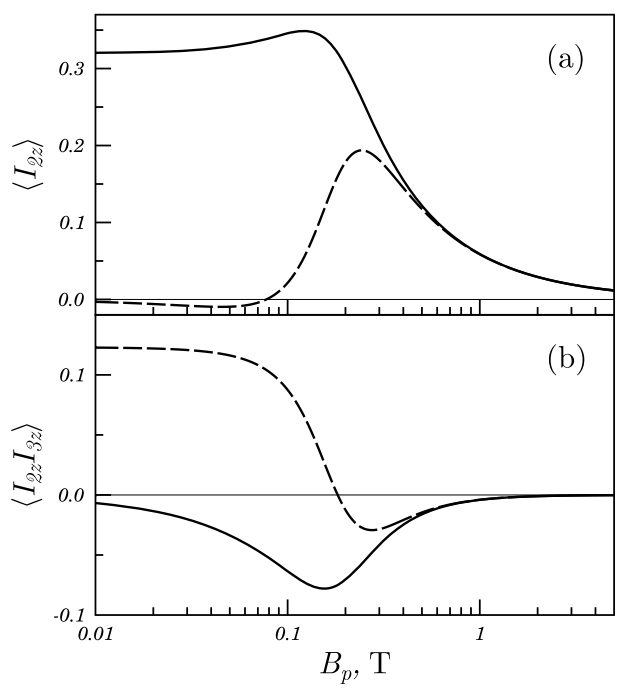

Fig. 3. Magnetic field dependence of PHIP of a 3-spin ABX-system for adiabatic (solid line) and sudden (dashed line) field variation. Here net PHIP of spins is shown $\left\langle I_{2 z}\right\rangle$ (a) and second-order multiplet effect $\left\langle I_{2 z} I_{3 z}\right\rangle$ (b). Only one of the net and multiplet polarizations is shown (in this case this is $\left\langle I_{2 z}\right\rangle$ and $\left\langle I_{2 z} I_{3 z}\right\rangle$; their counterparts $\left\langle I_{1 z}\right\rangle$ and $\left\langle I_{1 z} I_{3 z}\right\rangle$ differ only in sign). Multiplet PHIP $\left\langle I_{1 z} I_{2 z}\right\rangle$ is always equal to $-1 / 4$, third-order PHIP is zero. Parameters of the calculation: $\sigma_{1}=2 \mathrm{ppm}, \sigma_{2}=1 \mathrm{ppm}$, third spin is a heteronucleus, $J_{12}=5 \mathrm{~Hz}, J_{13}=15 \mathrm{~Hz}, J_{23}=3 \mathrm{~Hz}, \chi=\left(p_{\text {para }}-p_{\text {ortho }} / 3\right)=1$.

is in full agreement with Eqs. (27) and (29). The three-spin order (mutual entanglement of all three spins), $\left\langle I_{1 z} I_{2 z} I_{3 z}\right\rangle$, is always equal to zero. The field dependencies for polarization of the ABX-system are shown in Fig. 3. Interestingly, the multiplet HP of the X-spin, $\left\langle I_{1 z} I_{3 z}\right\rangle=-\left\langle I_{2 z} I_{3 z}\right\rangle$, drastically depends on the speed of field variation. For instance, at very low $B_{\mathrm{p}}$, at which the two Zeeman interactions, $v_{1}$ and $v_{2}$, are much smaller than the $J$-couplings (and the spin system becomes an $\mathrm{AA}^{\prime} \mathrm{X}$ system) multiplet polarization $\left\langle I_{1 z} I_{3 z}\right\rangle$ is expected only for sudden field switching. Not only the magnitude of the multiplet HP but even its sign is sensitive to the switching speed.

NMR spectra for different polarization fields are shown in Figs. 4 and 5. The PHIP spectra of the A and B spins (Fig. 4) exhibit both net and multiplet HP, whereas in the spectrum of the X-spin there is only multiplet HP present, which results in half of the lines with absorptive and half of the lines with emissive phase (Fig. 5). In principle, the NMR spectrum of the $\mathrm{X}$-spin consists of four lines; however, because of the relation for multiplet polarizations $\left\langle I_{1 z} I_{3 z}\right\rangle=-\left\langle I_{2 z} I_{3 z}\right\rangle$, two of them have zero intensity. As a consequence, there are only two lines in the PHIP spectra of the X-spin at any polarization field and field switching speed (Fig. 5).

Using the results for the ABX-system we can easily obtain the solution for the PHIP patterns of the AXB-system. This case is given when spins 1 and 2 (coming from para$\mathrm{H}_{2}$ ) form an AX-system, and one of them is strongly coupled to the third (initially non-polarized) spin in the molecule. In this case the eigen-states of the system can be obtained from Eqs. (21)-(22) by exchanging the second and the third spins. The state 
(a)
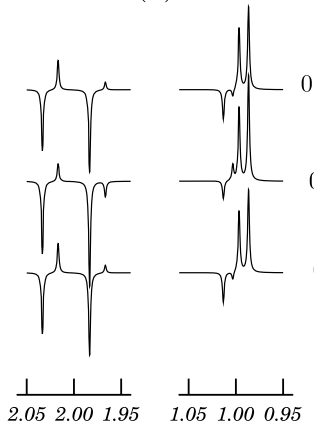

$\frac{\perp}{1.05} 1.00 \quad \frac{1.95}{0.95}$

$\sigma, \mathrm{ppm}$ (b)
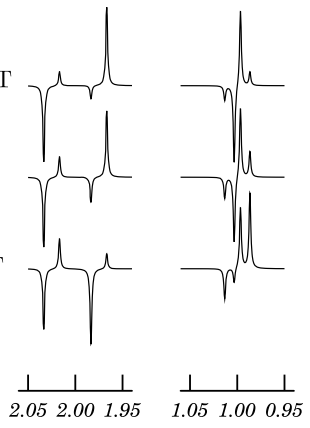

$\sigma, \mathrm{ppm}$

Fig. 4. PHIP spectra for a 3-spin $\mathrm{ABX}$-system; here the $\mathrm{AB}$-part of the spectrum is presented for adiabatic (a) and sudden (b) field variation for different polarization fields $(0.1 \mathrm{mT}, 0.1 \mathrm{~T}$ and $0.3 \mathrm{~T})$. For $0.1 \mathrm{mT}$ the spin system becomes AA'X-system. NMR parameters of the calculation are the same as for Fig. 3, pulse rotation angle is $\varphi=\pi / 4$.

(a)

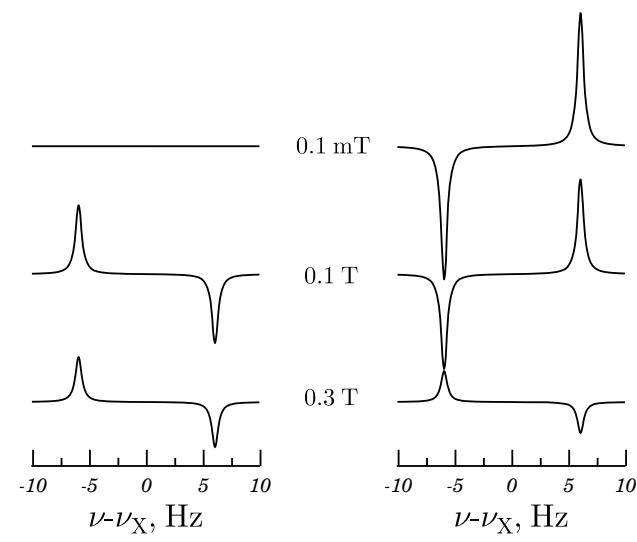

Fig. 5. PHIP spectra for a 3-spin ABX-system; here the X-part of the spectrum is presented for adiabatic (a) and sudden (b) field variation for different polarization fields $(0.1 \mathrm{mT}, 0.1 \mathrm{~T}$ and $0.3 \mathrm{~T})$. NMR parameters of the calculation are the same as for Fig. 3.

populations can be obtained in the same fashion:

$$
\begin{aligned}
& p_{1+}=0, \quad p_{2+}=\frac{\sin ^{2} \theta_{+}}{4}, \quad p_{3+}=\frac{\cos ^{2} \theta_{+}}{4}, \quad p_{4+}=\frac{1}{4} ; \\
& p_{1-}=\frac{1}{4}, \quad p_{2-}=\frac{\cos ^{2} \theta_{-}}{4}, \quad p_{3-}=\frac{\sin ^{2} \theta_{-}}{4}, \quad p_{4-}=0 .
\end{aligned}
$$

The way of calculating the expectation values of spin operators follows closely that for the ABX-system. For adiabatic field switching and $\sigma_{1}>\sigma_{2}$ the expressions take the 
form:

$$
\begin{aligned}
& \left\langle I_{1 z}\right\rangle=-\left\langle I_{3 z}\right\rangle=-\frac{\cos 2 \theta_{+}-\cos 2 \theta_{-}}{8}, \quad\left\langle I_{2 z}\right\rangle=0 ; \\
& \left\langle I_{1 z} I_{3 z}\right\rangle=0, \quad\left\langle I_{1 z} I_{2 z}\right\rangle=-\frac{1}{8}-\frac{\cos 2 \theta_{+}+\cos 2 \theta_{-}}{16}, \\
& \left\langle I_{2 z} I_{3 z}\right\rangle=-\frac{1}{8}+\frac{\cos 2 \theta_{+}+\cos 2 \theta_{-}}{16} ; \\
& \left\langle I_{1 z} I_{2 z} I_{3 z}\right\rangle=0 .
\end{aligned}
$$

For sudden field variation the expectation values are given by:

$$
\begin{aligned}
& \left\langle I_{1 z}\right\rangle=-\left\langle I_{3 z}\right\rangle=-\frac{\cos ^{2} 2 \theta_{+}-\cos ^{2} 2 \theta_{-}}{8}, \quad\left\langle I_{2 z}\right\rangle=0 \\
& \left\langle I_{1 z} I_{3 z}\right\rangle=0, \quad\left\langle I_{1 z} I_{2 z}\right\rangle=-\frac{1}{8}-\frac{\cos ^{2} 2 \theta_{+}+\cos ^{2} 2 \theta_{-}}{16}, \\
& \left\langle I_{2 z} I_{3 z}\right\rangle=-\frac{1}{8}+\frac{\cos ^{2} 2 \theta_{+}+\cos ^{2} 2 \theta_{-}}{16} \\
& \left\langle I_{1 z} I_{2 z} I_{3 z}\right\rangle=0 .
\end{aligned}
$$

These results can be understood in the following way. As one of the spins (X-spin) coming from para- $\mathrm{H}_{2}$ is only weakly coupled to the rest of the molecule (A- and Bspins $)$ it cannot acquire any net PHIP $\left(\left\langle I_{2 z}\right\rangle=0\right)$. Similarly, the total net polarization of the AB-system is equal to zero $\left(\left\langle I_{1 z}+I_{3 z}\right\rangle=0\right)$. Nevertheless, the spins constituting the AB-system acquire net HP of opposite sign; its amplitude is determined by the difference between $\theta_{+}$and $\theta_{-}$. Multiplet HP of the X-spin with the rest of the molecule, which is given by $\left\langle\left(I_{1 z}+I_{3 z}\right) I_{2 z}\right\rangle=\left(\left\langle I_{1 z} I_{2 z}\right\rangle+\left\langle I_{2 z} I_{3 z}\right\rangle\right)$, is equal to $-1 / 4$ (the same value of multiplet PHIP as for the AX-system). However, there is also a difference between $\left\langle I_{1 z} I_{2 z}\right\rangle$ and $\left\langle I_{3 z} I_{2 z}\right\rangle$. These results are qualitatively the same for sudden and adiabatic field switching. The behavior of such a system is similar to that of the simple AXsystem, in which the A spin "splits" into two subparts (A- and B-spins). Multiplet PHIP of these two "subparts" is zero as well as the three spin order $\left\langle I_{1 z} I_{2 z} I_{3 z}\right\rangle$.

Thus, as the main conclusion for the ABX-system we emphasize that spin-spin interaction of the AB-system with spin $\mathrm{X}$ may lead to its polarization. However, such polarization is of pure multiplet character. Only strong coupling of the spins 1 and 2 may lead to their net polarization.

As far as the applications of hetero-nuclear PHIP are concerned an important issue is the possibility of creating net polarization of hetero-nuclei. Such a type of hyperpolarization has a number of advantages: (i) it can be long-lived (when the hetero-nucleus has a low gyromagnetic ratio and therefore relaxes slowly) and (ii) the spectrum does not contain lines with opposite phases that cancel each other when the spectral resolution is low. The analytical solution of PHIP transfer from protons to hetero-nuclei (i.e., solving the Hamiltonian of the corresponding spin system) is no longer possible; therefore we performed numerical calculations of PHIP.

Now let us demonstrate the efficiency of spin order transfer from protons to net polarization of a spin $1 / 2$ hetero-nucleus. In contrast to the multiplet-type polarization of 


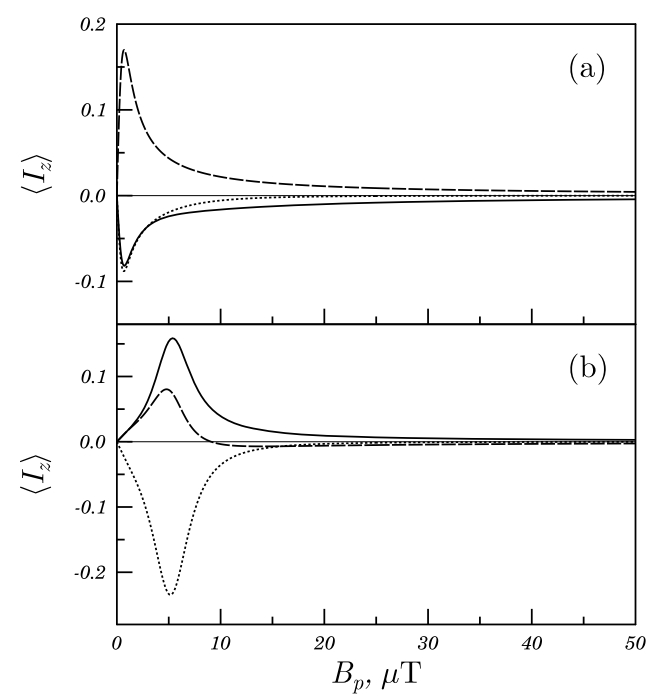

Fig. 6. Net polarization in the three-spin system as a function of magnetic field $B_{\mathrm{p}}$ in the case of directly polarized protons and a hetero-nucleus, which is ${ }^{13} \mathrm{C}$ (a) or ${ }^{19} \mathrm{~F}(\mathrm{~b})$. Polarization of the two protons is shown by solid and dashed lines; polarization of hetero-nucleus is shown by dotted lines. Parameters of calculation: $J_{12}=15 \mathrm{~Hz}, J_{13}=200 \mathrm{~Hz}, J_{23}=0$ (for subplot a); $J_{12}=15 \mathrm{~Hz}, J_{13}=7 \mathrm{~Hz}, J_{23}=0$ (for subplot b); $\chi=\left(p_{\text {para }}-p_{\text {ortho }} / 3\right)=1$, sudden field variation was taken.

hetero-nuclei, which can be formed up to relatively high fields, transfer of net polarization can occur only at very low fields. This is because of the requirement that the spins of protons and hetero-nuclei are strongly coupled. At such low fields the difference in Zeeman interaction of the two protons with the external fields becomes negligible, hence the Hamiltonian of the system can be written as follows:

$$
\hat{H}_{\mathrm{ABC}}=2 \pi\left\{-v_{p}\left(\hat{I}_{1 z}-\hat{I}_{2 z}\right)-v_{h} \hat{I}_{3 z}+J_{12}\left(\hat{\mathbf{I}}_{1} \cdot \hat{\mathbf{I}}_{2}\right)+J_{13}\left(\hat{\mathbf{I}}_{1} \cdot \hat{\mathbf{I}}_{3}\right)+J_{23}\left(\hat{\mathbf{I}}_{2} \cdot \hat{\mathbf{I}}_{3}\right)\right\} .
$$

Here $v_{p}$ and $v_{h}$ are the Larmor precession frequencies of the protons and the heteronucleus, respectively. In order to describe the polarization transfer we calculated the density matrix of the polarized molecule from Eqs. (6)-(8) at different fields and calculated the net polarizations of all three spins, $\left\langle I_{i z}\right\rangle=\operatorname{Tr}\left\{\hat{I}_{i z} \hat{\sigma}\right\}$, as a function of $B_{\mathrm{p}}$ assuming sudden field variation from $B_{\mathrm{p}}$ to the detection field. The results of calculation are shown in Fig. 6 assuming that the hetero-nucleus is ${ }^{13} \mathrm{C}$ (Fig. 6a) and ${ }^{19} \mathrm{~F}$ (Fig. 6b). In both cases it is possible to polarize the hetero-nucleus to a very high extent by setting the proper magnetic field. However, in both cases the optimal field is relatively low (about a few $\mu \mathrm{T}$, which is at least ten times smaller than the Earth field). Nevertheless, such a field is still achievable enabling strong enhancements of ${ }^{13} \mathrm{C}$ or ${ }^{19} \mathrm{~F}$ NMR signals by means of PHIP. It is worth noting that although the difference in frequencies is about $6 \%$ of $v_{p}$ for protons and ${ }^{19} \mathrm{~F}$ and $75 \%$ of $v_{p}$ for protons and ${ }^{13} \mathrm{C}, J$-couplings of protons and hetero-nuclei, in particular with ${ }^{13} \mathrm{C}$ can reach rather high values (up to $100-200 \mathrm{~Hz}$ ), which leads to strong coupling, $\left(v_{p}-v_{h}\right)<\max \left\{J_{13}, J_{23}\right\}$, and, consequently, to polarization of both hetero-nuclei at comparable magnetic field strengths. 
When raising the field to the Earth's field $(\approx 50 \mu \mathrm{T})$ where usually the ALTADENA experiments are performed the polarization of hetero-nuclei considerably (by approximately three orders of magnitude) decreases. However, it is still noticeably higher than the thermal polarization.

Now let us turn to a system of three coupled protons; the analytical solution of such a case is possible only for fields so low that the entire system is strongly coupled. This situation is discussed in Sect. 3.3.

\subsection{Three-spin system of type $\mathbf{A A}^{\prime} \mathbf{A}^{\prime \prime}$ at the polarization field}

Here we consider the three-spin system when at the polarization field the difference between all $v_{i}$ is negligible ( $\mathrm{AA}^{\prime} \mathrm{A}^{\prime \prime}$-system). The case of the $\mathrm{AA}^{\prime} \mathrm{A}^{\prime \prime}$-system has to a large extent been solved by Vinogradov and Grant [16] who obtained expressions for the state populations at the polarization field considering adiabatic field variation. Here we will extend the consideration to the sudden case and, in addition, determine rules for the net and multiplet HP.

The Hamiltonian of the $\mathrm{AA}^{\prime} \mathrm{A}^{\prime \prime}$-system at the polarization field is as follows:

$$
\hat{H}_{A A^{\prime} A^{\prime \prime}}=2 \pi\left\{J_{12}\left(\hat{\mathbf{I}}_{1} \cdot \hat{\mathbf{I}}_{2}\right)+J_{13}\left(\hat{\mathbf{I}}_{1} \cdot \hat{\mathbf{I}}_{3}\right)+J_{23}\left(\hat{\mathbf{I}}_{2} \cdot \hat{\mathbf{I}}_{3}\right)\right\} .
$$

Here, all Zeeman interactions are omitted because for each pair of spins $i, j$ the quantity $\left|v_{i}-v_{j}\right|$ is much smaller than the $J$-couplings The eigen-states of the $\mathrm{AA}^{\prime} \mathrm{A}^{\prime \prime}$ spin system and their energies are [17]:

$$
\begin{array}{ll}
|1\rangle=|\alpha \alpha \alpha\rangle, & E_{1}=\Sigma / 4 \\
|2\rangle=\frac{|\alpha \alpha \beta\rangle+|\alpha \beta \alpha\rangle+|\beta \alpha \alpha\rangle}{\sqrt{3}}, & E_{2}=E_{1}=\Sigma / 4 \\
|3,4\rangle=c_{1}^{ \pm}|\alpha \alpha \beta\rangle+c_{2}^{ \pm}|\alpha \beta \alpha\rangle+c_{3}^{ \pm}|\beta \alpha \alpha\rangle, & E_{3,4}=-\Sigma / 4 \pm \Lambda / 2 \\
|5,6\rangle=c_{3}^{ \pm}|\alpha \beta \beta\rangle+c_{2}^{ \pm}|\beta \alpha \beta\rangle+c_{1}^{ \pm}|\beta \beta \alpha\rangle, & E_{5,6}=E_{3,4}=-\Sigma / 4 \pm \Lambda / 2 \\
|7\rangle=\frac{|\alpha \beta \beta\rangle+|\beta \alpha \beta\rangle+|\beta \beta \alpha\rangle}{|8\rangle=|\beta \beta \beta\rangle,} & E_{7}=E_{1}=\Sigma / 4 \\
\mid 8 / 4 & E_{8}=E_{1}=\Sigma / 4
\end{array}
$$

where

$$
\Sigma=J_{12}+J_{13}+J_{23}, \quad \Lambda=\sqrt{\Sigma^{2}-3 \Gamma^{2}}, \quad \Gamma^{2}=J_{12} J_{13}+J_{12} J_{23}+J_{13} J_{23}
$$

The coefficients $c_{i}^{ \pm}$are as follows:

$$
\begin{aligned}
& c_{1}^{ \pm}=-\left(J_{12}-J_{23} \pm \Lambda\right) / \Theta^{ \pm}, \quad c_{2}^{ \pm}=\left(J_{13}-J_{23} \pm \Lambda\right) / \Theta^{ \pm}, \\
& c_{3}^{ \pm}=\left(J_{12}-J_{13}\right) / \Theta^{ \pm},
\end{aligned}
$$

where

$$
\Theta^{ \pm}=\sqrt{\left(J_{12}-J_{13}\right)^{2}+\left(J_{12}-J_{23} \pm \Lambda\right)^{2}+\left(J_{13}-J_{23} \pm \Lambda\right)^{2}}
$$


State populations have been obtained first by Vinogradov and Grant [16]. In our notation they are as follows:

$$
\begin{aligned}
& p_{1}=p_{2}=p_{7}=p_{8}=0, \quad p_{3}=p_{5}=\frac{1}{4}\left(c_{2}^{+}-c_{3}^{+}\right)^{2}, \\
& p_{4}=p_{6}=\frac{1}{4}\left(c_{2}^{-}-c_{3}^{-}\right)^{2}
\end{aligned}
$$

In the adiabatic case for states with fixed $I_{z}$ value the lowest state at the polarization field must be correlated with that of lowest energy at the detection field, the second lowest at $B=B_{\mathrm{p}}$ with the second lowest at $B=B_{0}$ and so on. We shall perform such a correlation for the case of all positive $J$-couplings with $\sigma_{1}>\sigma_{2}>\sigma_{3}$. In this case there is the following relation for the energies at the polarization field

$$
E_{2}>E_{3}>E_{4}, \quad E_{7}>E_{5}>E_{6}
$$

and at the detection field:

$$
E_{\alpha \alpha \beta}>E_{\alpha \beta \alpha}>E_{\beta \alpha \alpha}, \quad E_{\alpha \beta \beta}>E_{\beta \alpha \beta}>E_{\beta \beta \alpha} .
$$

State populations at the detection field are found accordingly:

$$
\begin{aligned}
& p_{\alpha \alpha \beta}=p_{\alpha \beta \beta}=0, \quad p_{\alpha \beta \alpha}=p_{\beta \alpha \beta}=\frac{1}{4}\left(c_{2}^{+}-c_{3}^{+}\right)^{2}, \\
& p_{\beta \alpha \alpha}=p_{\beta \beta \alpha}=\frac{1}{4}\left(c_{2}^{-}-c_{3}^{-}\right)^{2} .
\end{aligned}
$$

This allows us to find the following net magnetizations:

$$
\left\langle I_{1 z}\right\rangle=-\left\langle I_{3 z}\right\rangle=-\frac{\left(c_{2}^{-}-c_{3}^{-}\right)^{2}}{4}, \quad\left\langle I_{2 z}\right\rangle=0 .
$$

The second-order multiplet polarizations are:

$$
\left\langle I_{1 z} I_{2 z}\right\rangle=-\frac{\left(c_{2}^{+}-c_{3}^{+}\right)^{2}}{8}=\left\langle I_{2 z} I_{3 z}\right\rangle, \quad\left\langle I_{1 z} I_{3 z}\right\rangle=\frac{\left(c_{2}^{+}-c_{3}^{+}\right)^{2}}{8}-\frac{\left(c_{2}^{-}-c_{3}^{-}\right)^{2}}{8} .
$$

Finally, the third-order multiplet effect is zero: $\left\langle I_{1 z} I_{2 z} I_{3 z}\right\rangle=0$.

Correlation of states in other cases (different sign of $J$-couplings and differences in chemical shifts) is done correspondingly (will not be shown here). In any case, we are able to formulate the following rules. First, the spin with the second largest chemical shift will acquire only multiplet polarization. Second, the spin with the largest chemical shift in the $\sigma$-scale will acquire negative net PHIP, while that with the smallest chemical shift will have positive polarization of the same magnitude. Such a simple shape of the low-field PHIP (and CIDNP) pattern suggests that it follows from a more general rule applicable to multi-spin systems [38]. Due to the long preparation time PHIP is completely mixed among all three spins and, therefore, transferred efficiently to spin 3 , which initially had no spin order. When $\Sigma$ becomes negative, the energy levels at low 
field are rearranged in such a way (namely, $E_{3,5}>E_{4,6}>E_{2,7}$ ) that all signs of polarization introduced in Eqs. (43) and (44) change. This effect is similar to that observed for the AB-system, where the sign of net PHIP also changes upon sign inversion of the spin-spin interaction. In general, phases and magnitudes of PHIP crucially depend on the particular values of the $J$-couplings.

For the sudden field variation we calculate the expectation values at the polarization field, which gives us the following results for the net polarizations

$$
\left\langle I_{1 z}\right\rangle=\left\langle I_{2 z}\right\rangle=\left\langle I_{3 z}\right\rangle=0,
$$

the second-order multiplet polarizations

$$
\begin{aligned}
& \left\langle I_{1 z} I_{2 z}\right\rangle=\frac{\left(c_{2}^{+}-c_{3}^{+}\right)^{2}\left(2\left(c_{1}^{+}\right)^{2}-1\right)+\left(c_{2}^{-}-c_{3}^{-}\right)^{2}\left(2\left(c_{1}^{-}\right)^{2}-1\right)}{8}, \\
& \left\langle I_{1 z} I_{3 z}\right\rangle=\frac{\left(c_{2}^{+}-c_{3}^{+}\right)^{2}\left(2\left(c_{2}^{+}\right)^{2}-1\right)+\left(c_{2}^{-}-c_{3}^{-}\right)^{2}\left(2\left(c_{2}^{-}\right)^{2}-1\right)}{8}, \\
& \left\langle I_{2 z} I_{3 z}\right\rangle=\frac{\left(c_{2}^{+}-c_{3}^{+}\right)^{2}\left(2\left(c_{3}^{+}\right)^{2}-1\right)+\left(c_{2}^{-}-c_{3}^{-}\right)^{2}\left(2\left(c_{3}^{-}\right)^{2}-1\right)}{8}
\end{aligned}
$$

and $\left\langle I_{1 z} I_{2 z} I_{3 z}\right\rangle=0$. Zero net PHIP can be explained by the fact that for the $\mathrm{AA}^{\prime} \mathrm{A}^{\prime \prime}-$ system there is no preferred axis in space so that all orientations of the spins are equally probable. The reason that in the case of adiabatic field variation net HP is observed is the result of the spin dynamics during the slow field variation $B_{\mathrm{p}} \rightarrow B_{0}$. For sudden switching this dynamics has no time to occur, hence the spins keep the HP formed at the polarization field. Thus, net polarization observed for $B_{\mathrm{p}}=0$ indicates that field variation is sufficiently slow. On the other hand, one should be careful when interpreting low-field PHIP experiments, because the standard ALTADENA analysis is valid only when field switching to the high observation field is adiabatic. However, for any speed of field switching one acquires multiplet polarization.

Using our analytical formulas we have calculated PHIP spectra of the AA'A"system. The NMR parameters model the three spin-system in the styrene molecule, which is obtained from ethylbenzene by hydrogenation of the triple $\mathrm{C}-\mathrm{C}$ bond. The calculated spectra are shown in Fig. 7 for the two limiting cases of field variation. In the case of adiabatic field switching (Fig. 7, spectrum 1) the spins with the largest and smallest chemical shifts exhibit net polarization of opposite sign, whereas the spin with second highest chemical shift has zero net polarization. In the situation of sudden field variation (Fig. 7, spectrum 2) only multiplet spin order is formed. In addition, we have made a numerical simulation of the PHIP pattern with a field variation profile that is realistic for the experimental setup [23,24] available at the Free University of Berlin with the total switching time $\tau_{\mathrm{fv}}=0.41 \mathrm{~s}$. The corresponding PHIP spectrum (Fig. 7, spectrum 3) exhibits only a minor difference relative to that for the adiabatic field variation. This is a clear indication that under realistic conditions for the spin system considered the field switching provides almost adiabatic changes of the Hamiltonian (1). To check this expectation we made a comparison of the theoretical predictions with the PHIP spectrum of styrene (Fig. 7, spectrum 4) [48] taken at low field of $5 \mathrm{mT}$ and the 


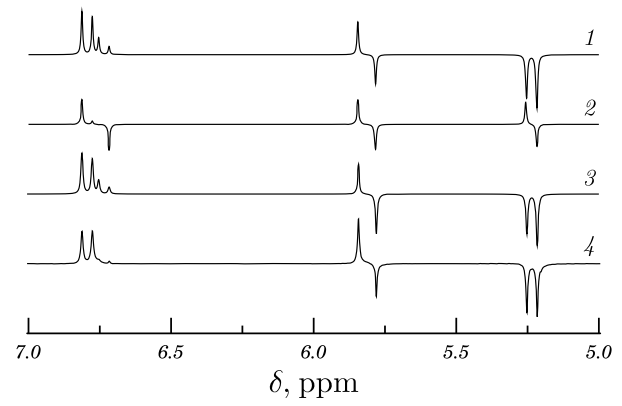

Fig. 7. PHIP spectra of a three-spin $\mathrm{AA}^{\prime} \mathrm{A}^{\prime \prime}$-system at $B_{\mathrm{p}}$ for adiabatic (spectrum 1) and sudden (spectrum 2) field switching and realistic field variation profile with $\tau_{\mathrm{fv}}=0.41 \mathrm{~s}$ (spectrum 3 ). NMR parameters of the three-spin system: $\delta_{1}=5.237 \mathrm{ppm}, \delta_{2}=6.674 \mathrm{ppm}, \delta_{3}=5.815 \mathrm{ppm}-$ chemical shifts; $J_{12}=$ $1.05 \mathrm{~Hz}, J_{13}=10.9 \mathrm{~Hz}, J_{23}=17.7 \mathrm{~Hz}-J$-couplings; pulse rotation angle $\varphi=\pi / 4$. Experimental PHIP spectrum of styrene (spectrum 4 ) taken at $B_{\mathrm{p}}=5 \mathrm{mT}, \varphi=\pi / 4$ and $\tau_{\mathrm{fv}}=0.41 \mathrm{~s}$ is also shown for comparison.

same field variation time, $\tau_{\mathrm{fv}}=0.41 \mathrm{~s}$, and pulse rotation angle, $\varphi=\pi / 4$, as in the calculation. The small $B_{\mathrm{p}}$ value guarantees that the spin system is of the $\mathrm{AA}^{\prime} \mathrm{A}^{\prime \prime}$-type at the preparation field. The experimental spectrum is in very good agreement with the theoretically calculated spectra for adiabatic switching (spectrum 1) and realistic field variation profile (spectrum 3).

Thus, both the analytical results for adiabatic field variation and the numerical calculation for a realistic field variation profile show good agreement with the experimental data. Finally, let us consider in Sect. 3.4 another special case of the three-spin system that can be solved analytically, namely, the $\mathrm{A}_{2} \mathrm{~B}$-system.

\subsection{Three-spin system of type $A_{2} B$ at the polarization field}

Here we consider the $\mathrm{A}_{2} \mathrm{~B}$-system, where the first A-spin and the B-spin come from the para $-\mathrm{H}_{2}$ molecule (born in singlet state), while the third spin is equivalent to the first one. As we always assign the numbers 1 and 2 to the spins of the para- $\mathrm{H}_{2}$ molecule, it is more accurate to use the term ABA-system. The Hamiltonian of the ABA-system at the polarization field is as follows:

$$
\hat{H}_{\mathrm{ABA}}=2 \pi\left\{-v_{1} \hat{I}_{1 z}-v_{2} \hat{I}_{2 z}-v_{1} \hat{I}_{3 z}+J_{12}\left(\hat{\mathbf{I}}_{1} \cdot \hat{\mathbf{I}}_{2}\right)+J_{12}\left(\hat{\mathbf{I}}_{2} \cdot \hat{\mathbf{I}}_{3}\right)\right\} .
$$

It is relatively simple as the two $J$-couplings and two of the chemical shifts are identical. It can be diagonalized analytically because not only $\left\langle I_{z}\right\rangle$ of all three spins is preserved but also the total spin of nucleus 1 and 3 (equal to 0 or 1 ) is a good quantum number. The eigen-states of the ABA-system are as follows:

$$
\begin{aligned}
& |1\rangle=|\alpha \alpha \alpha\rangle \\
& |2\rangle=\frac{|\alpha \alpha \beta\rangle-|\beta \alpha \alpha\rangle}{\sqrt{2}}, \quad|3\rangle=\cos \psi_{+}|\alpha \beta \alpha\rangle+\sin \psi_{+} \frac{|\alpha \alpha \beta\rangle+|\beta \alpha \alpha\rangle}{\sqrt{2}},
\end{aligned}
$$




$$
\begin{aligned}
|4\rangle & =-\sin \psi_{+}|\alpha \beta \alpha\rangle+\cos \psi_{+} \frac{|\alpha \alpha \beta\rangle+|\beta \alpha \alpha\rangle}{\sqrt{2}}, \\
|5\rangle & =\frac{|\alpha \beta \beta\rangle-|\beta \beta \alpha\rangle}{\sqrt{2}}, \quad|6\rangle=\cos \psi_{-}|\beta \alpha \beta\rangle+\sin \psi_{-} \frac{|\alpha \beta \beta\rangle+|\beta \beta \alpha\rangle}{\sqrt{2}}, \\
|7\rangle & =-\sin \psi_{-}|\beta \alpha \beta\rangle+\cos \psi_{-} \frac{|\alpha \beta \beta\rangle+|\beta \beta \alpha\rangle}{\sqrt{2}}, \\
|8\rangle & =|\beta \beta \beta\rangle .
\end{aligned}
$$

The magnetic field dependent mixing angles, $\psi_{+}$and $\psi_{-}$, are as follows:

$$
\psi_{ \pm}=\frac{1}{2} \arctan \left(\frac{J_{12} \sqrt{2}}{ \pm\left(\sigma_{1}-\sigma_{2}\right) v_{p}-J_{12} / 2}\right)
$$

State populations at the polarization field can be found in the same way as previously and are of the form:

$$
\begin{aligned}
& p_{1}=p_{8}=0, \quad p_{2}=p_{5}=\frac{1}{8}, \\
& p_{3}=\frac{1+\cos ^{2} \psi_{+}}{8}-\frac{\sin 2 \psi_{+}}{4 \sqrt{2}}, \quad p_{4}=\frac{1+\sin ^{2} \psi_{+}}{8}+\frac{\sin 2 \psi_{+}}{4 \sqrt{2}}, \\
& p_{6}=\frac{1+\cos ^{2} \psi_{-}}{8}-\frac{\sin 2 \psi_{-}}{4 \sqrt{2}}, \quad p_{7}=\frac{1+\sin ^{2} \psi_{-}}{8}+\frac{\sin 2 \psi_{-}}{4 \sqrt{2}},
\end{aligned}
$$

To obtain the expectation values of HP in the case of adiabatic field variation one can again perform correlation of the spin eigen-states at polarization and detection field. We assume that at the detection field $\left|v_{1}^{0}-v_{2}^{0}\right| \gg J_{12}$, i.e., the spin system is an AXAsystem, and $\sigma_{1}>\sigma_{2}$. Then the correlation of states is as follows:

$$
\begin{aligned}
& |3\rangle \rightarrow|\alpha \beta \alpha\rangle, \quad|4\rangle \rightarrow \frac{|\alpha \alpha \beta\rangle+|\beta \alpha \alpha\rangle}{\sqrt{2}}, \\
& |6\rangle \rightarrow \frac{|\alpha \beta \beta\rangle+|\beta \beta \alpha\rangle}{\sqrt{2}}, \quad|7\rangle \rightarrow|\beta \alpha \beta\rangle
\end{aligned}
$$

For AXA-system states $|2\rangle$ and $|4\rangle$ become degenerate as well as states $|5\rangle$ and $|7\rangle$ do. Attributing the populations from Eq. (50) to the high field states according to Eq. (51) we obtain the following expression for the net HP:

$$
\left\langle I_{1 z}\right\rangle=\left\langle I_{3 z}\right\rangle=-\frac{\left\langle I_{2 z}\right\rangle}{2}=\frac{p_{3}-p_{7}}{2}=\frac{\cos 2 \psi_{+}+\cos 2 \psi_{-}}{32}-\frac{\sin 2 \psi_{+}+\sin 2 \psi_{-}}{8 \sqrt{2}} .
$$

Multiplet HP is given by the following equation:

$$
\begin{aligned}
\left\langle I_{1 z} I_{2 z}\right\rangle & =\left\langle I_{2 z} I_{3 z}\right\rangle=-\frac{p_{3}+p_{7}}{4} \\
& =-\frac{3}{32}-\frac{\cos 2 \psi_{+}-\cos 2 \psi_{-}}{64}+\frac{\sin 2 \psi_{+}-\sin 2 \psi_{-}}{16 \sqrt{2}} .
\end{aligned}
$$




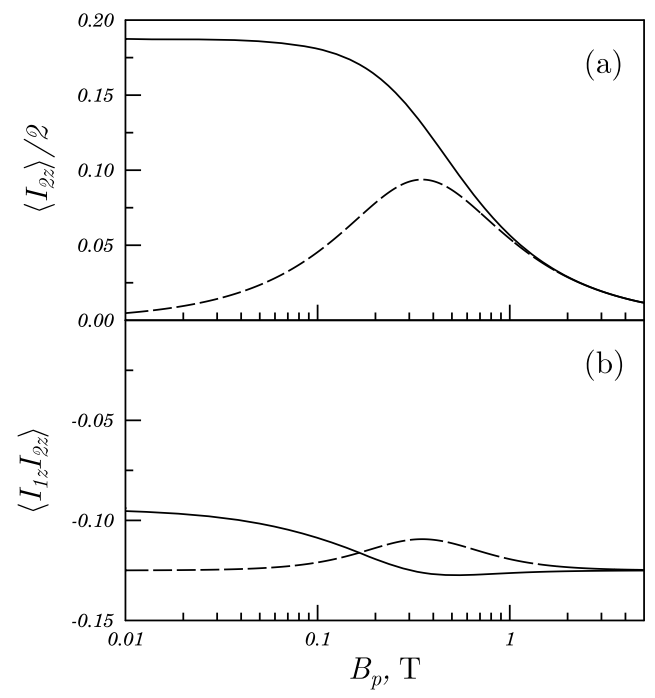

Fig. 8. Magnetic field dependence of PHIP of an ABA-system for adiabatic (solid lines) and sudden (dashed lines) field variation. Here net PHIP of spins $\left\langle I_{2 z}\right\rangle$ (a) and second-order multiplet effect $\left\langle I_{1 z} I_{2 z}\right\rangle$ (b) are shown. Parameters of the calculation: $\sigma_{1}=\sigma_{3}=2 \mathrm{ppm}, \sigma_{2}=1 \mathrm{ppm}, J_{12}=J_{23}=10 \mathrm{~Hz}$, $\chi=\left(p_{\text {para }}-p_{\text {ortho }} / 3\right)=1$.

In the case of sudden field variation correlation of states is not required and the quantities of interest (expectation values of the corresponding operators) are calculated straightforwardly as the expectation values at the polarization field. As a result,

$$
\begin{aligned}
& \left\langle I_{1 z}\right\rangle=\left\langle I_{3 z}\right\rangle=-\frac{\left\langle I_{2 z}\right\rangle}{2}=\frac{p_{3} \cos ^{2} \psi_{+}+p_{4} \sin ^{2} \psi_{+}-p_{6} \cos ^{2} \psi_{-}-p_{7} \sin ^{2} \psi_{-}}{2} ; \\
& \left\langle I_{1 z} I_{2 z}\right\rangle=\left\langle I_{2 z} I_{3 z}\right\rangle=-\frac{p_{3} \cos ^{2} \psi_{+}+p_{4} \sin ^{2} \psi_{+}+p_{6} \cos ^{2} \psi_{-}+p_{7} \sin ^{2} \psi_{-}}{4} .
\end{aligned}
$$

The full expressions with the explicit form of $p_{i}$ given are rather cumbersome. In the case of the ABA-system there is no need to discuss the multiplet HP of the type $\left\langle I_{1 z} I_{3 z}\right\rangle$ (multiplet HP of the two A-spins) because it does not reveal itself in the spectra of the AXA-system. Only when the spins 1 and 3 become non-equivalent there will be extra lines in the spectrum, which exhibit multiplet HP of such a type. The intensities of these lines will depend on $\left\langle I_{1 z} I_{3 z}\right\rangle$, however, for the ABA-system they overlap in the spectrum, hence all contributions of the $\left\langle I_{1 z} I_{3 z}\right\rangle$ polarization exactly compensate each other. The three-spin order, $\left\langle I_{1 z} I_{2 z} I_{3 z}\right\rangle$, is equal to zero.

Field dependencies for the ABA-system are shown in Fig. 8. As previously, a strong dependence of PHIP on the field switching speed is expected. For $B_{\mathrm{p}}=0$ and the adiabatic case (ALTADENA conditions) non-zero net HP is observable, while for the sudden case at $B_{\mathrm{p}}=0$ net HP is, obviously, zero. At high $B_{\mathrm{p}}$ net HP goes to zero in any case, while at intermediate field strength it is non-zero in either case. Multiplet HP (Fig. 8) does not show any pronounced field dependence, and its limiting value (for adiabatic and sudden field variation) at high field is equal to $-1 / 8$. 


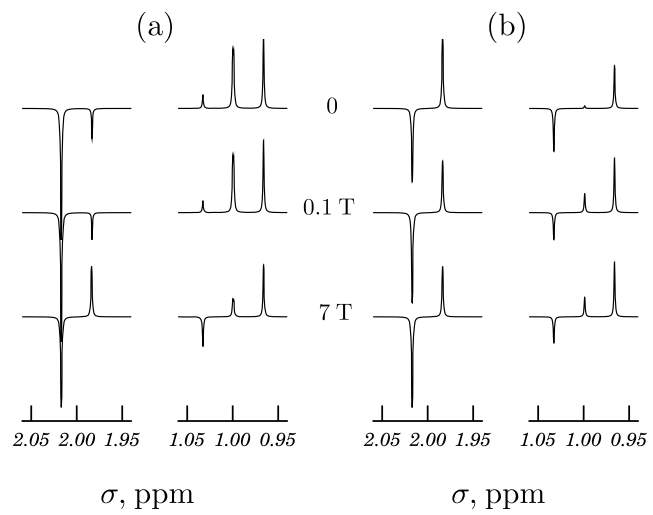

Fig. 9. PHIP spectra of a 3-spin ABA-system at different $B_{\mathrm{p}}$ for adiabatic (a) and sudden (b) field variation. NMR parameters of the calculation are the same as for Fig. 6, pulse rotation angle $\varphi=\pi / 4$.

PHIP spectra calculated for both switching regimes and different polarization field strength are shown in Fig. 9. At low fields they strongly depend on the speed of field variation. However, at high fields they exactly coincide, since both net and multiplet PHIP are the same for the sudden and adiabatic cases (see Fig. 9). Interestingly, for $B_{\mathrm{p}}=0$ and sudden field switching the spectrum is similar to the PASADENA spectrum. The reason for this is the same as for the AB-system. It comes from the fact that neither any net HP is formed at $B_{\mathrm{p}}=0$ because of the isotropy of space, nor the spins can acquire it during fast field variation. Another similarity to the spin systems considered above is that for adiabatic field variation the spin with largest chemical shift $\sigma_{i}$ always acquires negative net HP. However, when the sign of $J$-couplings $\left(J_{12}\right.$ and $\left.J_{23}\right)$ becomes negative the net PHIP of both spins changes sign.

\section{Summary and conclusions}

In the present article we have described the details of a recently developed theoretical approach to PHIP phenomena. In particular, we focused here on obtaining analytical results for PHIP of a few model spin systems. These systems are of $\mathrm{AB}, \mathrm{ABX}, \mathrm{AA}^{\prime} \mathrm{A}^{\prime \prime}$ and ABA character, for which the spin Hamiltonian can be diagonalized analytically. This allowed us obtaining compact formulas for the expectation values of the spin operators and the field dependence of polarization. Only dynamic evolution of the polarized spin system under the influence of $J$-couplings has been considered, whereas relaxation effects have been completely neglected. In general, variation of the coupling regime (from strong coupling at low field to weak coupling at high fields) is the key factor responsible for the PHIP pattern and PHIP re-distribution in the molecule. We restricted the treatment to the situation of long PHIP preparation periods so that only non-equilibrium eigen-state populations are formed, whereas the coherences between them are zero. In general, the evolution of coherences is an important factor for multispin systems as it enables efficient PHIP re-distribution in the molecule. We have also considered effects of field switching on the polarization patterns, which is an important issue in PHIP 
experiments. The two limiting cases of very slow (adiabatic) and very fast (sudden) switching were considered.

As follows from our study the speed of field switching strongly affects the spectral pattern. As a result, for instance, the spectral pattern obtained when polarizing at low field $B_{\mathrm{p}}$ and switching suddenly from $B_{\mathrm{p}}$ to $B_{0}$ is of the PASADENA type instead of the ALTADENA type because the adiabaticity condition is not fulfilled. Such effects have not been observed so far, presumably, because field variation, which is done manually in most ALTADENA experiments, is not fast enough. Our previous experiments on PHIP of styrene, which have been found to be sensitive to the field variation speed [21], confirm this statement. From our present study we can also conclude that in case of adiabatic field variation and positive $J$-coupling all ALTADENA spectra exhibit similar features: spins with larger chemical shifts (in the $\sigma$-scale) acquire negative net polarization, while those with smaller chemical shifts carry positive net HP (total net PHIP is zero). When the spin-spin coupling is negative the phase of the spectrum is opposite.

Our analytical results allow us formulating PHIP sign rules for ALTADENA experiments by analogy with Kaptein's rules for CIDNP [49]. In this context, by the ALTADENA case we imply that the preparation field, $B_{\mathrm{p}}$, is so low that the frequency differences, $v_{i}-v_{j}$, are negligible at this field. We will not formulate rules for multiplet polarization because for a two-spin system it is obviously equal to $-1 / 4$, whereas for higher spin systems the PHIP pattern is too complex for establishing any simple sign rules. The same holds for the multiplet polarization of a larger number of spins, for instance, for $\left\langle I_{i z} I_{j z} I_{k z}\right\rangle$ and higher spin orders. Summarizing our analytical results we can write down the following rule for the sign, $\Gamma_{i}$, of net PHIP of the $i$-th nucleus:

$$
\Gamma_{i}=\Gamma_{\sigma} \Gamma_{J} \Gamma_{H} \Gamma_{\mathrm{fc}} .
$$

Here $\Gamma_{i}=+: A, \Gamma_{i}=0, \Gamma_{i}=-1: E$, stand for the absorptive, zero and emissive net polarization of the $i$-th nucleus. The quantities on the right-hand side should be read as follows. $\Gamma_{\sigma}$ is the sign of $\left(\sigma_{i}-\langle\sigma\rangle\right)$ with $\langle\sigma\rangle$ being the average chemical shift of the group of spins: spins with the highest and lowest chemical shifts acquire PHIP of opposite sign, while the spins in the center of the NMR spectrum tend to have zero net polarization. When the $\delta$-scale of chemical shifts is used $-\Gamma_{\delta}=\left(\langle\delta\rangle-\delta_{i}\right)$ must be taken instead. $\Gamma_{J}$ is the sign of the effective spin-spin interaction, which is equal to $J_{12}$ for the AB-system and to $\Sigma$ for the AA'A"-system. For systems with more coupled spins it can be defined in a similar way; in most cases $\Gamma_{J}$ should be set to +1 . $\Gamma_{H}$ is a factor describing the predominant spin state of the $\mathrm{H}_{2}$ molecule prior to the hydrogenation (para or ortho spin isomer):

$$
\Gamma_{H}= \begin{cases}-1, & \text { para; } \\ +1, & \text { ortho }\end{cases}
$$

Typically $\Gamma_{H}$ is set to -1 as it is easy to enrich $\mathrm{H}_{2}$ gas in its para-component. The last factor, $\Gamma_{\mathrm{fc}}$, stands for the field switching regime:

$$
\Gamma_{\mathrm{fc}}= \begin{cases}1, & \text { adiabatic } \\ 0, & \text { sudden }\end{cases}
$$


The rule Eq. (55) thus gives a simple description of the ALTADENA spectral pattern. We expect that these rules are valid for most of the spin systems although in some cases they can be violated by complex evolution in multi-spin systems just as well as Kaptein's rules may not always hold [50].

However, as the switching turns into the non-adiabatic regime the appearance of the spectrum changes. As a consequence, it becomes essential to use the actual field variation profile for obtaining good agreement between simulation and experimental data. PHIP reveals itself not only in net HP of spins but, most notably, in multiplet polarization, which represents their entanglement. It is particularly worth mentioning that it possible to polarize hetero-nuclear spins by means of PHIP by creating multiplet polarization of the hetero-nucleus and protons. In contrast to net polarization of hetero-nuclei (which requires going to very low magnetic fields) such polarization can be formed at much higher fields, which are much easier accessible. PHIP spectra are sensitive not only to the speed of field variation but also to the strength of the polarization field. Controlling the polarization field strength and the field switching profile allows one to manipulate the PHIP spectral pattern and optimize the relative size of different spin orders. In addition, it opens new possibilities in transferring polarization selectively to certain target spins of choice in a controllable way. It is important to mention that in many cases it is advantageous to perform PHIP at intermediate field strengths for getting higher signal-to-noise ratio. Besides this, PHIP-based polarizers operating at such fields can be designed as portable stand-alone devices for in vivo or in situ imaging [51].

For modeling PHIP spectra of simple 2-spin and 3-spin systems our analytical results can be directly applied. If the coupled system contains a higher number of spins and the relation between the quantities $\left|v_{1}-v_{2}\right|$ and the spin-spin interactions, $J_{i j}$, is arbitrary the Hamiltonian of the system can no longer be solved analytically. As a consequence, for more complex systems numerical calculations are required, which can be carried out in the framework of our theoretical approach [20,21]. Systems of three and more spins have the interesting feature that they have level anti-crossings at distinctive magnetic fields. In the vicinity of such a level crossing there is an efficient coherent polarization exchange of HP among the coupled spins [30,33]. Precise field variation involving level anti-crossing fields enables transferring polarization in a selective and controllable way.

\section{Acknowledgement}

Financial support by the Russian Fund for Basic Research (RFBR project No. 1103-00356, 11-03-00296), Program of the Division of Chemistry and Material Science RAS (project No. 5.1.1) and the Program P-220 of the Russian Government (grant No. 11.G34.31.0045) is gratefully acknowledged. We acknowledge support from the Research Group Linkage Program of the Alexander von Humboldt Foundation. We are thankful to Dr. Alexey Kiryutin (Freie Universität Berlin) for providing us with an experimental ALTADENA spectrum of styrene and to the reviewer who suggested to formulate simple rules for $\Gamma_{i}$. 


\section{References}

1. K. M. Salikhov, Y. N. Molin, R. Z. Sagdeev, and A. L. Buchachenko, Spin Polarization and Magnetic Effects in Chemical Reactions, Elsevier, Amsterdam (1984).

2. W. Happer, Rev. Mod. Phys. 44 (1972) 169.

3. B. M. Goodson, J. Magn. Reson. 155 (2002) 157.

4. D. Stehlik and H.-M. Vieth, Mol. Cryst. Liq. Cryst. 93 (1983) 83.

5. D. Stehlik and H.-M. Vieth, Time Evolution of Electron-Nuclear Cross-Polarization in Radiofrequency Induced Optical Nuclear Spin Polarization, in: Pulsed Magnetic Resonance: NMR, ESR, and Optics: A Recognition of E. L. Hahn, D. M. S. Bagguley (Ed.), Oxford University Press, Oxford (1992), p. 446.

6. C. D. Jeffries, Dynamic Nuclear Orientation, Interscience, New York (1963).

7. K. H. Hausser and D. Stehlik, Adv. Magn. Reson. 3 (1968) 79.

8. M. Borghini, W. De Boer, and K. Morimoto, Phys. Lett. A 48A (1974) 244.

9. J. Natterer and J. Bargon, Prog. NMR Spectrosc. 31 (1997) 293.

10. S. B. Duckett and C. J. Sleigh, Prog. NMR Spectrosc. 34 (1999) 71.

11. K. Muennemann and H. W. Spiess, Nat. Phys. 7 (2011) 522.

12. R. W. Adams, J. A. Aguilar, K. D. Atkinson, M. J. Cowley, P. I. P. Elliott, S. B. Duckett, G. G. R. Green, I. G. Khazal, J. Lopez-Serrano, and D. C. Williamson, Science 323 (2009) 1708.

13. M. G. Pravica and D. P. Weitekamp, Chem. Phys. Lett. 145 (1988) 255.

14. C. R. Bowers and D. P. Weitekamp, J. Am. Chem. Soc. 109 (1987) 5541.

15. S. Bouguet-Bonnet, F. Reineri, and D. Canet, J. Chem. Phys. 130 (2009) 234507.

16. E. Vinogradov and A. K. Grant, J. Magn. Reson. 194 (2008) 46.

17. H. Jóhannesson, O. Axelsson, and M. Karlsson, C. R. Phys. 5 (2004) 315.

18. S. Aime, R. Gobetto, F. Reineri, and D. Canet, J. Magn. Reson. 178 (2006) 184.

19. L. Buljubasich, M. B. Franzoni, H. W. Spiess, and K. Münnemann, J. Magn. Reson. 219 (2012) 33.

20. K. L. Ivanov, A. V. Yurkovskaya, and H.-M. Vieth, J. Chem. Phys. 128 (2008) 154701.

21. S. E. Korchak, K. L. Ivanov, A. V. Yurkovskaya, and H.-M. Vieth, Phys. Chem. Chem. Phys. 11 (2009) 11146.

22. J. Natterer, T. Greve, and J. Bargon, Chem. Phys. Lett. 293 (1998) 455.

23. S. Grosse, F. Gubaydullin, H. Scheelken, H.-M. Vieth, and A. V. Yurkovskaya, Appl. Magn. Reson. 17 (1999) 211.

24. S. Grosse, A. V. Yurkovskaya, J. Lopez, and H.-M. Vieth, J. Phys. Chem. A 105 (2001) 6311.

25. T. Theis, P. Ganssle, G. Kervern, S. Knappe, J. Kitching, M. P. Ledbetter, D. Budker, and A. Pines, Nat. Phys. 7 (2011) 571.

26. T. Theis, M. P. Ledbetter, G. Kervern, J. W. Blanchard, P. J. Ganssle, M. C. Butler, H. D. Shin, D. Budker, and A. Pines, J. Am. Chem. Soc. 134 (2012) 3987.

27. B. C. Hamans, A. Andreychenko, A. Heerschap, S. S. Wijmenga, and M. Tessari, J. Magn. Reson. 212 (2011) 224.

28. S. Gloeggler, R. Mueller, J. Colell, M. Emondts, M. Dabrowski, B. Bluemich, and S. Appelt, Phys. Chem. Chem. Phys. 13 (2011) 13759.

29. Q. Gong, A. Gordji-Nejad, B. Bluemich, and S. Appelt, Anal. Chem. 82 (2010) 7078.

30. K. Miesel, K. L. Ivanov, A. V. Yurkovskaya, and H.-M. Vieth, Chem. Phys. Lett. 425 (2006) 71.

31. K. L. Ivanov, K. Miesel, A. V. Yurkovskaya, S. E. Korchak, A. S. Kiryutin, and H.-M. Vieth, Appl. Magn. Reson. 30 (2006) 513.

32. S. Schäublin, A. Wokaun, and R. R. Ernst, Chem. Phys. 14 (1976) 285.

33. K. Miesel, K. L. Ivanov, T. Koechling, A. V. Yurkovskaya, and H.-M. Vieth, Appl. Magn. Reson. 34 (2008) 423.

34. R. R. Ernst, G. Bodenhausen, and A. Wokaun, Principles of Nuclear Magnetic Resonances in One and Two Dimensions, Clarendon Press, Oxford (1978).

35. S. Schäublin, A. Wokaun, and R. R. Ernst, J. Magn. Reson. 27 (1977) 273. 
36. S. Schäublin, A. Höhener, and R. R. Ernst, J. Magn. Reson. 13 (1974) 196.

37. J.-K. Vollenweider and H. Fischer, Chem. Phys. 108 (1986) 365.

38. K. L. Ivanov, K. Miesel, H.-M. Vieth, A. V. Yurkovskaya, and R. Z. Sagdeev, Z. Phys. Chem. 217 (2003) 1641.

39. V. F. Tarasov and I. A. Shkrob, J. Magn. Reson. Ser. A 109 (1994) 65.

40. K. L. Ivanov, H.-M. Vieth, K. Miesel, A. V. Yurkovskaya, and R. Z. Sagdeev, Phys. Chem. Chem. Phys. 5 (2003) 3470.

41. G. Buntkowsky, J. Bargon, and H.-H. Limbach, J. Am. Chem. Soc. 118 (1996) 8677.

42. M. Haake, J. Barkenmeyer, and J. Bargon, J. Phys. Chem. 99 (1995) 17539.

43. L. T. Kuhn and J. Bargon, Top. Curr. Chem. 276 (2007) 25.

44. K. Golman, O. Axelsson, H. Jóhannesson, S. Mansson, C. Olofsson, and J. S. Petersson, Magn. Reson. Med. 46 (2001) 1.

45. L. T. Kuhn, U. Bommerich, and J. Bargon, J. Phys. Chem. A 110 (2006) 3521.

46. M. Roth, A. Koch, P. Kindervater, J. Bargon, H. W. Spiess, and K. Münnemann, J. Magn. Reson. 204 (2010) 50.

47. U. Bommerich, T. Trantzschel, S. Mulla-Osman, G. Buntkowsky, J. Bargon, and J. Bernarding, Phys. Chem. Chem. Phys. 12 (2010) 10309.

48. A. S. Kiryutin, private communication (2012).

49. R. Kaptein, Chem. Commun. (1971) 732.

50. K. M. Salikhov, Chem. Phys. 64 (1982) 371.

51. K. W. Waddell, A. M. Coffey, and E. Y. Chekmenev, J. Am. Chem. Soc. 133 (2011) 97. 